\title{
Oxygen Radical Functionalization of Boron Nitride Nanosheets
}

Toby Sainsbury ${ }^{1,2, *}$, Amro Satti ${ }^{1,3}$, Peter May ${ }^{1,2}$, Zhiming Wang ${ }^{1,2}$, Ignatious McGovern ${ }^{1,2}$, Yurii K. Gun'ko ${ }^{1,3}$, Jonathan Coleman ${ }^{1,2, \uparrow}$

${ }^{l}$ Centre for Research on Adaptive Nanostructures and Nanodevices (CRANN), Trinity College Dublin, Dublin 2, Ireland.

${ }^{2}$ School of Physics, Trinity College Dublin, Dublin 2, Ireland.

${ }^{3}$ School of Chemistry, Trinity College Dublin, Dublin 2, Ireland.

E-mail: *toby.sainsbury@gmail.com, ${ }^{\dagger}$ colemaj@tcd.ie

\begin{abstract}
The covalent chemical functionalization of exfoliated hexagonal boron-nitride nanosheets (BNNSs) is achieved by the solution phase oxygen radical functionalization of boron atoms in the h-BN lattice. This involves a two-step procedure to initially covalently graft alkoxy groups to boron atoms and the subsequent hydrolytic defunctionalisation of the groups to yield hydroxyl-functionalized BNNSs $(\mathrm{OH}-$ BNNSs). Characterization of the functionalized-BNNSs using HR-TEM, Raman, UV-Vis, FTIR, NMR, and TGA was performed to investigate both the structure of the BNNSs and the covalent functionalization methodology. OH-BNNSs were used to prepare polymer nanocomposites and their mechanical properties analyzed. The influence of the functional groups grafted to the surface of the BNNSs is investigated by demonstrating the impact on mechanical properties of both non-covalent and covalent bonding at the interface between the nanofiller and polymer matrices.
\end{abstract}

\section{Introduction}

Recent years have seen significant research attention directed towards the production of exfoliated graphene nano-sheets. ${ }^{1-3}$ Motivated by the potential application of individual graphene nanosheets for electronic, optical, mechanical reinforcement and catalytic applications, a deluge of research activity has investigated novel properties of this crystalline 2-dimensional material. ${ }^{4}$ These studies have in turn drawn attention to a range of analogous 2-dimensional layered materials such as transition metal dichalcogenides, perovskites, transition metal oxides and hexagonal boron-nitride (h-BN). ${ }^{5-7}$ These materials are known to possess a diverse suite of properties such as high thermal conductivity, mechanical strength, and a range of intrinsic electronic behaviours. ${ }^{5-7}$ Consequently, scalable liquid-phase exfoliation of 2-D layered materials such as graphene, $\mathrm{MoS}_{2}, \mathrm{WS}_{2}$, and h-BN to individual nanosheets has resulted in a large number of reports in the literature concerning exfoliation, solubility studies and the integration of such materials within host polymer systems to form composites. ${ }^{8-10}$ It is noted that multiple reports have described covalent chemical modification of graphene with organic moieties to impart chemical functionality and hence facilitate integration within polymer systems. ${ }^{11-13}$ In recent years it has become clear that the ability to chemically functionalize graphene to graphene oxide has massively expanded its potential for applications. ${ }^{4,14}$ It is also clear that having an alternative isostructural material which is white/transparent, insulating, 
chemically stable, mechanically strong, thermal conductive, and which may be isolated as exfoliated atomic sheets, may hold many advantages over the carbon analogue for certain applications such as optical components, surface coatings and transparent composites. It is therefore highly attractive to be able to chemically functionalize h-BN in an extensive manner so that the functionalized h-BN is suitable for use in an expanded range of applications. There have been no reports comparable to graphene chemistry demonstrating the extensive covalent functionalization of $h$ $\mathrm{BN}$ with organic species. The covalent functionalization of h-BN with organic molecules is expected to significantly alter the surface energy and hence the solubility of functionalized h-BN in common solvents and to dictate the nature of the interaction between the h-BN and molecular or condensed phase materials. In order to take advantage of the intrinsic mechanical and thermally conductive properties of $\mathrm{h}-\mathrm{BN}$ it is therefore of interest to graft organic functional molecules to the surface of the material which are closely matched in chemical nature to an intended host matrix whilst retaining the structural integrity of the h-BN lattice.

Hexagonal Boron-Nitride (h-BN) is an extremely attractive material for multiple technological applications due to its exceptional mechanical, thermal and electronic properties. ${ }^{15}$ In addition to the established thermal, lubricating and mechanical applications of h-BN in the bulk phase, the applications of nanoscale exfoliated h-BN include an expansive array of applications based on its intrinsic properties. ${ }^{16}$ These include mechanical composites, ${ }^{10 \mathrm{~b}}$ superhydrophobic surface coatings, ${ }^{17,18}$ substrates for device electronics, ${ }^{7}$ gas diffusion barrier-composites ${ }^{19}$ and catalyst supports. ${ }^{20}$ The envisaged opportunity to tailor the electronic and magnetic properties of h-BN by doping or surface functionalization therefore provides a strong impetus to develop the covalent chemistry of isolated h-BN nanosheets (BNNSs). ${ }^{21}$ Theoretically predicted engineering of the wide band-gap semiconducting properties of $\mathrm{h}$ $\mathrm{BN}(5.5 \mathrm{eV})$ as well as the predicted edge functionalization of BNNSs or boron-nitride nanoribbons (BNNRs) to yield magnetic nanosheets make h-BN especially attractive as a blank white canvas that is available for covalent manipulation. ${ }^{22,23}$

Hexagonal-BN exists as a layered structure of alternating Boron and Nitrogen atoms in a hexagonal lattice isostructural to the hexagonal lattice of $\mathrm{C}$ atoms in a graphitic lattice. ${ }^{15} \mathrm{In}$ h-BN, adjacent sheets differ in stacking sequences of atomic planes with respect to $\mathrm{C}$ atoms in graphite. In h-BN, electron deficient $\mathrm{B}$ atoms are located directly above or beneath corresponding electron rich $\mathrm{N}$ atoms, whereas in graphite, hexagons are offset and alternating $\mathrm{C}$ atoms lie above and beneath hexagon centres. The ionic nature of the $\mathrm{B}-\mathrm{N}$ bond results in $\mathrm{ABAB}$ stacking geometry and yields an interlayer bonding distance of $\sim 0.33 \mathrm{~nm}$ while the B-N bond length is $1.44 \AA \AA$. The interlayer attractive forces are mediated by pseudo-delocalized out-of-plane $\pi$ orbital and weak van der Waals interactions. This highly stable and mechanically strong structure exhibits extremely high chemically stability and resistance to oxidation until temperatures of over $800{ }^{\circ} \mathrm{C}$ thus making h-BN highly attractive for a range of applications in the bulk-phase where chemical stability and structural integrity are demanded. ${ }^{16,24 a-c}$ It is therefore particularly challenging to initiate chemical reactions involving atoms in the bulk h-BN lattice whilst retaining the intrinsic properties of the material. In view of the recent advances in the area of exfoliated 2D nanosheets, it has become apparent that for the application of exfoliated h-BN nanosheets, alternative chemical strategies will be necessary in order to integrate and utilize BNNSs in applications such as surface coatings and nanocomposites. 
A recent report has shown that by ultrasonication assisted hydrolysis of h-BN sheets that edge functionalization can be achieved. ${ }^{25}$ Hydrolysis of h-BN edges is postulated to occur by addition of hydroxide ions to B atoms and by protons to $\mathrm{N}$ atoms; however such edge functionalization imparts negligible chemical modification with respect to the bulk characteristics of the h-BN crystallites. Notably, this procedure was shown to result in the destruction of extended h-BN sheets to form nanoscale fragments. It is known that for any intended mechanical application of nanosheet materials that extended length scales are optimal for matrix reinforcement. ${ }^{26}$ Therefore, avoiding the destruction of the intrinsic bonding structure and mechanical properties of BNNSs is necessary for application within composites. Multiple reports have described non-covalent interactions between organic molecules, polymers, bio-molecules and nanoparticles and the surface of both boron-nitride nanotubes (BNNTs) and h-BN. ${ }^{27}$ The noncovalent functionalization of BNNTs and h-BN has been described using alkyl amine, alkyl phosphine and aromatic containing molecule and polymers. ${ }^{27 a, b, 28 a, b}$ The interactions in these cases are mediated by electron rich nitrogen and oxygen species interacting with electron deficient boron atoms and by $\pi-\pi$ interactions respectively. Boron atoms therefore readily act as Lewis acids to the corresponding electron containing Lewis bases. This feature provides a valuable indication that in the presence of highly reactive radical species, vacant $\mathrm{p}$ orbitals in boron atoms may provide a route to bond formation and hence covalent functionalization.

In the case of BNNTs, covalent functionalization has been described at sparse amine defects, at extensive plasma grafted amino groups and more recently by hydrogen peroxide induced hydroxyl groups by Zhi and co-workers. ${ }^{29 a b c}$ Notably in the latter case, mixing of BNNTs in hydrogen peroxide is possible to allow functionalization whereas in the case of h-BN, intrinsic hydrophobicity severely limits the ability to disperse h-BN in an aqueous medium. ${ }^{29 \mathrm{c}}$ This is a critical feature, which prevents reliable experiments to probe the aqueous based chemistry of $\mathrm{h}-\mathrm{BN}$ and provides a focus for the current work. Following the report of BNNTs functionalization by Zhi and co-workers, recent work, carried out using strongly oxidizing solutions under high temperature and pressure has shown that the surface chemistry of h-BN may be modified with hydrophilic functional groups. ${ }^{30}$ With this in mind, the functionalization of exfoliated h-BN nanosheets (BNNSs) with organic functional groups is a logical goal. Whereby chemical functionality grafted to the B atoms in the h-BN lattice of exfoliated BNNSs would dictate their dispersion within solvent systems and their integration with molecular and condensed phase materials.

(a)
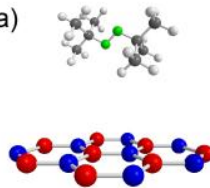

(d)

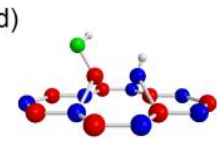

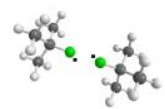
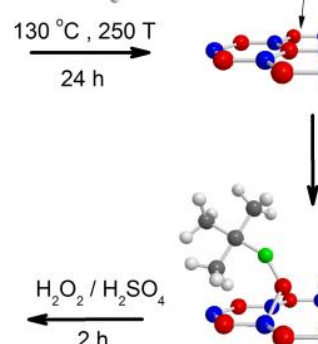

(c)

(b)
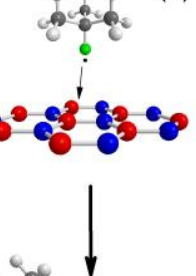

Scheme 1. Reaction methodology indicating (a) h-BN in the presence of di-tert-butyl peroxide, (b) tert-butoxy radical attack on a B atom in the h- 
BN lattice following thermolysis of the organic peroxide. (c) tert-butoxy

functionalized BNNSs (d) hydroxyl-functionalized BNNSs (OH-BNNSs).

In this work, we present a novel covalent functionalization approach for the oxidation of exfoliated h-BN nanosheets (BNNSs) to yield hydroxyl-functionalized-BNNSs (OH-BNNSs). This involves a two-step functionalization procedure. The first step involves the dispersion of solution exfoliated BNNSs within an organic peroxide, as depicted in Scheme 1, followed by the thermolysis of the peroxide to generate alkoxy radicals which functionalize B atoms in the h-BN lattice via oxygen radical attack (Scheme 1a-b). The second step involves removal of the BNNS-bound alkoxy groups via hydrolysis using a strongly oxidizing solution to yield surface bound hydroxyl groups, thus hydroxylated-BNNSs (OH-BNNSs), Scheme 1c-d. Hydroxyl functional groups are then used to integrate functionalized BNNSs within polymers based on specific interactions between functionalized BNNSs and the polymer matrices. A basis for the controlled compatibilization of BNNSs with diverse materials and substrates is thus envisaged as a result of this efficient functionalization strategy.

\section{Experimental}

\section{General}

Chemicals and reagents used were purchased from Sigma Aldrich and were used as received. Transmission Electron Microscopy (TEM) was conducted using a FEI, Titan (TM) 80-300 S/TEM operated at $80 \mathrm{kV}$ and a JEOL3000F operated at $100 \mathrm{kV}$. Samples for TEM were prepared by dropping a drop of a dilute suspension of h-BN onto a lacycarbon copper TEM grid and allowing the solvent to evaporate. Raman spectra were recorded using a Renishaw inVia Raman Microscope at $\lambda=514.5 \mathrm{~nm}$ laser excitation. Samples were prepared by evaporating exfoliated BNNSs onto gold coated silicon substrates for analysis of dispersed nanosheets. Absorption measurements were taken using a Varian Cary 6000i using a $1 \mathrm{~cm}$ quartz cuvette. FT-IR measurements were recorded using a Nicolet 6700, diamond ATR accessory. Thermogravimetric Analysis (TGA) was performed using a Perkin-Elmer Pyris-1 TGA system in air. The temperature was scanned from 25 to $900{ }^{\circ} \mathrm{C}$ at $10{ }^{\circ} \mathrm{C} / \mathrm{min}$. Sonication was carried out using Branson 2510EMT and Sonorex RK 1028H sonic baths and a VibraCell CVX; 750 W Sonic Tip. ${ }^{1}$ H-NMR spectra were recorded using a Bruker AV-400 Fourier Transform NMR spectrometer. Tensile testing was carried out using a Zwick proline tensile tester with a strain rate of $50 \mathrm{~mm} / \mathrm{min}$. X-Ray Photoelectron Spectroscopy (XPS) was performed in a system equipped with a VG CLAM II electron analyser and PSP twin anode source. Mg Ka (hv = $1253.6 \mathrm{eV}$ ) spectra were recorded at $10 \mathrm{eV}$ pass energy and $2 \mathrm{~mm}$ slits, yielding an overall resolution of $0.85 \mathrm{eV}$. Samples were prepared by evaporation of BNNS material from solution onto gold-coated Si-wafer substrates. Samples were introduced via a load-lock and a measurement base pressure was better than $10^{-9} \mathrm{mbar}$.

\section{Exfoliation of Boron-Nitride Nanosheets (BNNSs)}

Exfoliated BNNSs were prepared according to a recently published procedure. ${ }^{10 a}$ Briefly, this involved the sonication of h-BN in $N$-methyl 2-pyrrolidone (NMP) $(50 \mathrm{~mL})$ at a concentration of $3 \mathrm{mg} / \mathrm{mL}$ in a round-bottomed flask $(100 \mathrm{~mL})$ for $48 \mathrm{H}$. The suspension was centrifuged at $1500 \mathrm{rpm}$ for $45 \mathrm{mins}$ to remove any aggregated material. The suspension of exfoliated h-BN nanosheets (BNNSs) was left to equilibrate for $24 \mathrm{~h}$ to allow any insoluble material or aggregates to precipitate. The translucent milky white supernatant fraction was retained. 


\section{Optical Characterization}

UV-Vis analysis was performed in order to asses the modification of the solubility of the BNNSs following functionalization. In order to analyse the solubility and hence scattering behaviour of the h-BN material in solution, pristine BNNSs and TB-BNNSs were sonicated in propanol at a concentration of $3 \mathrm{mgmL}^{-1}$ using a sonic bath (Sonorox RK 1028H) for 24 hours to ensure optimum dispersion of the exfoliated materials. The materials were centrifuged at $1500 \mathrm{rpm}$ for 45 minutes in order remove any aggregated material and the supernatent fraction was retained in each case. The concentration of the suspensions was determined by taking an aliquot of the suspended material $(10 \mathrm{~mL})$ and filtering it using an Whatman-Anodisc, alumina filter membrane (pore size $20 \mathrm{~nm}$ ). The filter was dried using a vacuum oven $\left(40{ }^{\circ} \mathrm{C}\right)$ for $6 \mathrm{~h}$ and was then weighed to accurately determine the mass of the material retained. UV-Vis spectra were recorded for each of the dispersions and the absorbance value at a known wavelength, $300 \mathrm{~nm}$, determined.

\section{Preparation of tert-Butoxy-Functionalized Boron-Nitride Nanosheets (TB-BNNSs)}

Functionalization of the BNNSs by the tert-butoxy radical species involved the dispersion of the BNNSs in the liquid di-tert-butylperoxide reagent in a sealed high pressure autoclave vessel and the subsequent thermolysis of the peroxide at elevated temperature. The generation of the oxygen radicals amongst the exfoliated BNNSs ensures optimal conditions whereby the oxygen radicals can attack the exfoliated sheets. To carry out the oxygen radical functionalization of the BNNSs, the translucent milky white solution $(20 \mathrm{~mL})$ of the exfoliated BNNSs in NMP was transferred to the di-tert-butylperoxide (TBP) liquid reagent to allow the functionalization within the undiluted liquid peroxide reagent. This was achieved by filtration of the BNNSs using $20 \mathrm{~nm}$ Alumina filters followed by washing, re-suspension by sonication and re-washing using copious quantities of ethanol ( $200 \mathrm{~mL})$ and chloroform (200 L) relative to the mass of BNNSs ( 2 mg). The washed BNNSs on the Alumina filter membrane were analysed using Diamond-ATR-FTIR to ensure that no trace of residual NMP solvent remained within the BNNSs. The BNNSs material was then sonicated directly off the filter membrane into the clear TBP liquid $(4 \mathrm{~mL})$ in a round-bottomed flask. Since filtering the BNNSs may have allowed loose re-stacking of the BNNSs, the BNNSs were bath-sonicated for $12 \mathrm{~h}$ in the TBP to ensure optimal dispersion of the sheets and exposure to the neat liquid reagent. This should allow the maximum possible exposure of the BNNSs to the TB radicals and ensure optimum functionalization. The translucent milky white dispersion of BNNSs was then transferred to the Teflon inner jacket of a High Pressure Reactor Vessel (Autoclave). The reactor vessel was sealed and the heated to $120{ }^{\circ} \mathrm{C}$ for $12 \mathrm{~h}$ allowing the thermolysis of the TBP and subsequent functionalization of the BNNSs. Following the reaction, the reactor vessel was allowed to cool to room temperature and was opened to reveal a yellow-orange cloudy dispersion of h-BN material which gave off a sweet odour with a hint of acetic acid. Filtration of the liquid using a $20 \mathrm{~nm}$ Alumina membrane filter allowed the separation of a clear yellow-orange liquid by-product from the white h-BN material. Recovery of the functionalized h-BN following the reaction involved an extensive washing procedure whereby, on the alumina filter, the h-BN material was washed with ethanol $(300 \mathrm{~mL})$ followed by chloroform (300 $\mathrm{mL})$. The material was then sonicated into ethanol $(300 \mathrm{~mL})$ for $30 \mathrm{mins}$ and was re-filtered, followed by additional ethanol $(300 \mathrm{~mL})$ and chloroform $(300 \mathrm{~mL})$ wash to ensure complete re-dispersion of the material and separation of 
any residual reaction by-products or contaminants between sheets. This procedure was repeated twice more to complete the washing procedure.

\section{Preparation of Hydroxyl-Functionalized Boron-Nitride Nanosheets (OH-BNNSs)}

Hydrolysis of the organic tert-butoxy groups grafted to the surface of the BNNS (TB-BNNSs) was achieved by using the potent oxidizer; piranha solution $\left(\mathrm{H}_{2} \mathrm{SO}_{4}: \mathrm{H}_{2} \mathrm{O}_{2}, 3: 1\right)$, to yield hydroxyl groups bound to the boron atoms in the BNNS lattice, OH-BNNSs. Briefly, this involved, the addition of TB-BNNSs (10 mg) to concentrated sulphuric acid $(70 \%$ soln., $15 \mathrm{~mL})$ in a round-bottomed flask $(50 \mathrm{~mL})$ followed by bath sonication $(45 \mathrm{~min})$ to ensure optimum dispersion of the nanosheets. Under slow magnetic stirring, freshly opened hydrogen peroxide solution $(28 \%, 5 \mathrm{~mL})$ was then slowly added to the sulphuric acid-BNNS suspension. Following the final addition of the peroxide, the solution became vigorously exothermic accompanied by the rapid generation of oxygen and presumably carbon dioxide bubbles. The suspension was stirred for 2 hours until the evolution of bubbles had dissipated and the liquid was sufficiently cool for filtration. The material was then vacuum-filtered on an alumina membrane filter $(20 \mathrm{~nm})$ and washed with water $(2 \mathrm{~L})$. The material was sonicated off the filter into water $(1 \mathrm{~L})$ for 10 mins and filtered once again. This procedure was repeated once more to ensure any water-soluble residue was removed. This was followed by washing with propanol $(500 \mathrm{~mL})$ and chloroform $(500 \mathrm{~mL})$ to ensure the removal of any organic residue. The dry OH-BNNSs material on the filter was dispersed once again in water $(500 \mathrm{~mL})$ and sonicated (10 mins) and filtered a final time on an alumina membrane filter $(20 \mathrm{~nm})$. The OH-BNNS material on the filter was dried using a vacuum oven for $48 \mathrm{~h}$.

\section{Preparation of Isocyanate-Functionalized BNNSs (iBNNSs)}

Isocyanate-functionalized BNNSs (iBNNSs) were prepared by the carbamate-mediated linkage of the diisocyanate molecule, 1,6-hexamethylenediisocyanate, to hydroxyl-functionalized BNNSs (OH-BNNSs). This involved the addition of OH-BNNSs $(5 \mathrm{mg})$ to a two-necked round-bottomed flask $(50 \mathrm{~mL})$ which was purged of moisture/oxygen using Schlenk apparatus in conjunction with dry argon. 1,6-Hexamethylenediisocyanate (15 ml) added to the flask under argon, and the mixture was sonicated using a bath sonicator for $4 \mathrm{~h}$ to disperse the $\mathrm{OH}$ BNNS material. The pale/translucent reaction mixture was then heated to $65{ }^{\circ} \mathrm{C}$ for $24 \mathrm{~h}$ under magnetic stirring, under argon, and was then allowed to cool to room temperature. The iBNNS suspension was then filtered using membrane filtration apparatus (Millipore) in conjunction with an alumina filter membrane (Whatman Anodisc 0.200 $\mu \mathrm{m})$ by anhydrous N,N-dimethylformamide (DMF)(300 mL) followed by anhydrous dichloromethane (DCM)(300 $\mathrm{mL}$ ) in order to wash the iBNNS material from residual diisocyanate. The iBNNS material was then sonicated into anhydrous DCM (200 mL) and sonicated for 30 mins to disperse the iBNNS material and was filtered once again using DMF $(200 \mathrm{~mL})$ and DCM $(400 \mathrm{~mL})$. The extensive washing procedure ensures that any residual started diisocyanate molecules or reaction by-products are removed from the iBNNS material. The iBNNS material was then transferred by sonication from the filter membrane into DCM $(10 \mathrm{~mL})$ to a round bottomed flask $(10 \mathrm{~mL})$, evaporated to dryness using a rotary evaporator and was dried using Schlenk apparatus until used.

\section{Composite Preparation}

Polyvinylalcohol (PVA) composites; BNNS:PVA and OH-BNNS:PVA were prepared by dispersion of the dry 
BNNS material in water $(19 \mathrm{~mL})$ by sonication for $4 \mathrm{~h}$. PVA $\left(1 \mathrm{~g}, \mathrm{M}_{\mathrm{w}} 31-50 \mathrm{~K}\right)$ was added over $6 \mathrm{~h}$ to the vigorously stirring suspensions of BNNSs in each case to yield a 5\% solution, as well as to water for a blank PVA solution. This ensures that the filler material is optimally dispersed in the solvent and is then incrementally populated by the polymer chains as each pellet of PVA is added. The BNNS:PVA suspensions were then vigorously stirred for $72 \mathrm{~h}$ to ensure complete mixing. The composite solutions were then allowed to equilibrate for $48 \mathrm{~h}$ by standing. An aliquot of the blank and composite solutions $(1 \mathrm{~mL})$ was pipetted onto glass microscope slides and were allowed dry in a laboratory refrigerator $\left(T=\sim 3-5{ }^{\circ} \mathrm{C}\right)$ for $24 \mathrm{~h}$. The samples were further dried of residual solvent by drying in a vacuum oven for $48 \mathrm{~h}$ at $40{ }^{\circ} \mathrm{C}$. Samples were peeled off the glass substrates and were cut into test strips of $2.25 \mathrm{~mm}$ in width using a die cutter. Average thickness of the samples was $20-25 \mu \mathrm{m}$. We note that all samples were stored in ambient conditions before measurement. This results in water uptake to an equilibrium value. As a result the samples are more ductile and less stiff than is found for dry PVA-based samples.

Polyurethane (PU) composites; BNNS:PU and iBNNS:PU were prepared by dispersion of the dry BNNS material in anhydrous $\mathrm{N}, \mathrm{N}$-dimethylformamide $(\mathrm{DMF})(20 \mathrm{~mL})$ by sonication for $4 \mathrm{~h}$. PU, polyhexamethylene diisocyanate $(1$ g) $\left(M_{w} 70-100 \mathrm{~K}\right)$ was added over $6 \mathrm{~h}$ to the vigorously stirring suspensions of BNNSs in each case to yield the 5\% solutions of PU:BNNSs, as well as a blank PU solution. The BNNS:PU suspensions were then vigorously stirred for $72 \mathrm{~h}$ to ensure complete mixing. The composite solutions were then allowed to equilibrate for $48 \mathrm{~h}$ by standing. An aliquot of the blank and composite solutions $(1 \mathrm{~mL})$ was pipetted onto glass microscope slides and were allowed dry in a laboratory refrigerator $\left(\mathrm{T}=\sim 3-5^{\circ} \mathrm{C}\right)$ for $24 \mathrm{~h}$. The samples were further dried of residual solvent by drying in a vacuum oven for $48 \mathrm{~h}$ at $40{ }^{\circ} \mathrm{C}$. Samples were peeled off the glass substrates and were cut into test strips of $2.25 \mathrm{~mm}$ in width using a die cutter. Average thickness of the samples was 20-25 $\mu \mathrm{m}$.

\section{Results and Discussion}

The intrinsic chemical robustness of $\mathrm{h}-\mathrm{BN}$ is demonstrated by its resistance to oxidation and covalent chemical functionalization. ${ }^{24}$ This feature highlights the difficulty in attempting to chemically integrate h-BN with molecular and condensed phase materials by initiating chemical reactions with atoms in the h-BN lattice. The treatment of hBN in strongly oxidative conditions such as boiling aqua-regia, hot piranha solution, perchloric acid-potassium permanganate solution or refluxing nitric acid have been attempted by the authors to achieve oxidation without success. Given the hydrophobic characteristic of $\mathrm{h}-\mathrm{BN},{ }^{29 \mathrm{c}}$ it is not surprising that the aqueous-based oxidizing species have had limited success in interacting with the surface of h-BN to achieve oxidation. In order to achieve the oxidative functionalization of h-BN we used an organic peroxide, di-tert-butylperoxide (TBP), to disperse exfoliated BNNSs within the organic medium. Subsequent thermolysis of the peroxide to form tert-butoxy radicals capable of reacting with the surface of the $\mathrm{h}-\mathrm{BN}$ nanosheets was performed in order to form boronate ester C-O-B bonds to B atoms in the lattice. Therefore, the functionalization of BNNSs with the tert-butoxy groups is the first step in creating a chemical handle at the surface of the BNNSs. Tert-butoxy groups bound to the surface of the h-BN nanosheets are then susceptible to hydrolysis which would result in the creation of functional chemical handles which would significantly influence the surface energy of the BNNSs and importantly which would be capable of further chemical derivitization. Piranha solution, acidified hydrogen peroxide, known as a highly aggressive oxidizer 
for the decomposition of organic materials, was used to hydrolyze the surface-bound tert-butoxy groups from surface of the h-BN sheets to yield hydroxyl groups at B atoms in the B-N lattice and therefore creating hydroxylfunctionalized BNNSs (OH-BNNSs).

Prior to the organic functionalization procedure, in order to ensure an optimal surface area is available to the organic peroxide, we employed an exfoliation protocol recently reported by this group. ${ }^{10 a}$ By sonicating h-BN in $N$ methylpyrollidinone (NMP), followed by centrifugation and recovery of the supernatant fraction, h-BN was solution exfoliated (see experimental section). This procedure results in the production of mono- and multi-layer sheets from the bulk h-BN crystallites and thus provides a mixture of BNNS substrates for solution-based chemistry. Following recent reports describing the sensitivity of Raman signals to characterize interlayer effects. ${ }^{31 a, b}$ Relative to the principle interlayer Raman active $\mathrm{E}_{2 \mathrm{~g}}$ mode at $\sim 1366 \mathrm{~cm}^{-1}$, mono- and multi-layered h-BN sheets have been characterized by identification of red-shifting of the $\mathrm{E}_{2 \mathrm{~g}}$ band as bulk $\mathrm{h}-\mathrm{BN}$ exfoliates to multi-layers and a blue-shift of the order of $\sim 4 \mathrm{~cm}^{-1}$ upon exfoliation to monolayer h-BN. We use this technique in this work to track the exfoliation using our procedure. The procedure used follows a methodology recently reported to yield exfoliated h$\mathrm{BN}$ nanosheets and is described in the experimental section. During a $48 \mathrm{~h}$ exfoliation procedure, $(10 \mathrm{~mL})$ aliquots of the $\mathrm{h}-\mathrm{BN}$ suspension were sampled at $12 \mathrm{~h}$ and $24 \mathrm{~h}$ and upon completion at $48 \mathrm{~h}$. Samples for Raman analysis were performed on dispersed nanosheets dried from suspension onto gold substrates. Figure 1a shows the Raman spectra of the exfoliated h-BN. Initially for bulk h-BN, the $\mathrm{E}_{2 \mathrm{~g}}$ mode for bulk $\mathrm{h}-\mathrm{BN}$ is centred at $1366 \mathrm{~cm}^{-1}$, at $12 \mathrm{~h}$ exfoliation the band has red-shifted to $1364 \mathrm{~cm}^{-1}$ which we attribute to the formation of predominantly multi-layered material, and following $24 \mathrm{~h}$, the band is centred at $1365 \mathrm{~cm}^{-1}$ indicating the formation of predominantly bi/tri-layer structures. Extended exfoliation for a further $24 \mathrm{~h}$ was found to result in the $\mathrm{E}_{2 \mathrm{~g}}$ phonon mode centred at approximately $1370 \mathrm{~cm}^{-1}$, a blue-shift of $\sim 4 \mathrm{~cm}^{-1}$. This behaviour is explained by the elongation of B-N bonds in bulk h-BN due to interlayer interactions which leads to a softening of the phonons, relative to monolayer h-BN where the influence of interlayer interactions is absent and the B-N bond is expected to be shortened. ${ }^{31,32}$ These interactions between adjacent sheets have been postulated to comprise of bond iconicity of the B-N bond in the $\mathrm{D}_{6 \mathrm{~h}}$ point group symmetry configuration of alternating sheets in addition to weak orbital overlap interactions and van der Waal forces. ${ }^{31}$ Following exfoliation, the band at $1370 \mathrm{~cm}^{-1}$ has an average FWHM value of $9.5 \mathrm{~cm}^{-1}$ while in the starting h-BN material at $1366 \mathrm{~cm}^{-1}$ has an average FWHM value of $21.6 \mathrm{~cm}^{-1}$. This substantial change is attributed to a decrease in the superposition of multiple peaks which make up the $1366 \mathrm{~cm}^{-1}$ peak in h-BN in comparison to a more homogenous distribution of nanosheets with smaller sheet size following exfoliation and centrifugation (Additional statistics from multiple traces are provided in the Supporting Information (Figure S1)). The observed differences of the phonon frequency of the $\mathrm{E}_{2 \mathrm{~g}}$ mode before and following the exfoliation procedure supports the assertion that mono-layer and few-layer BNNSs are formed following the exfoliation protocol. 

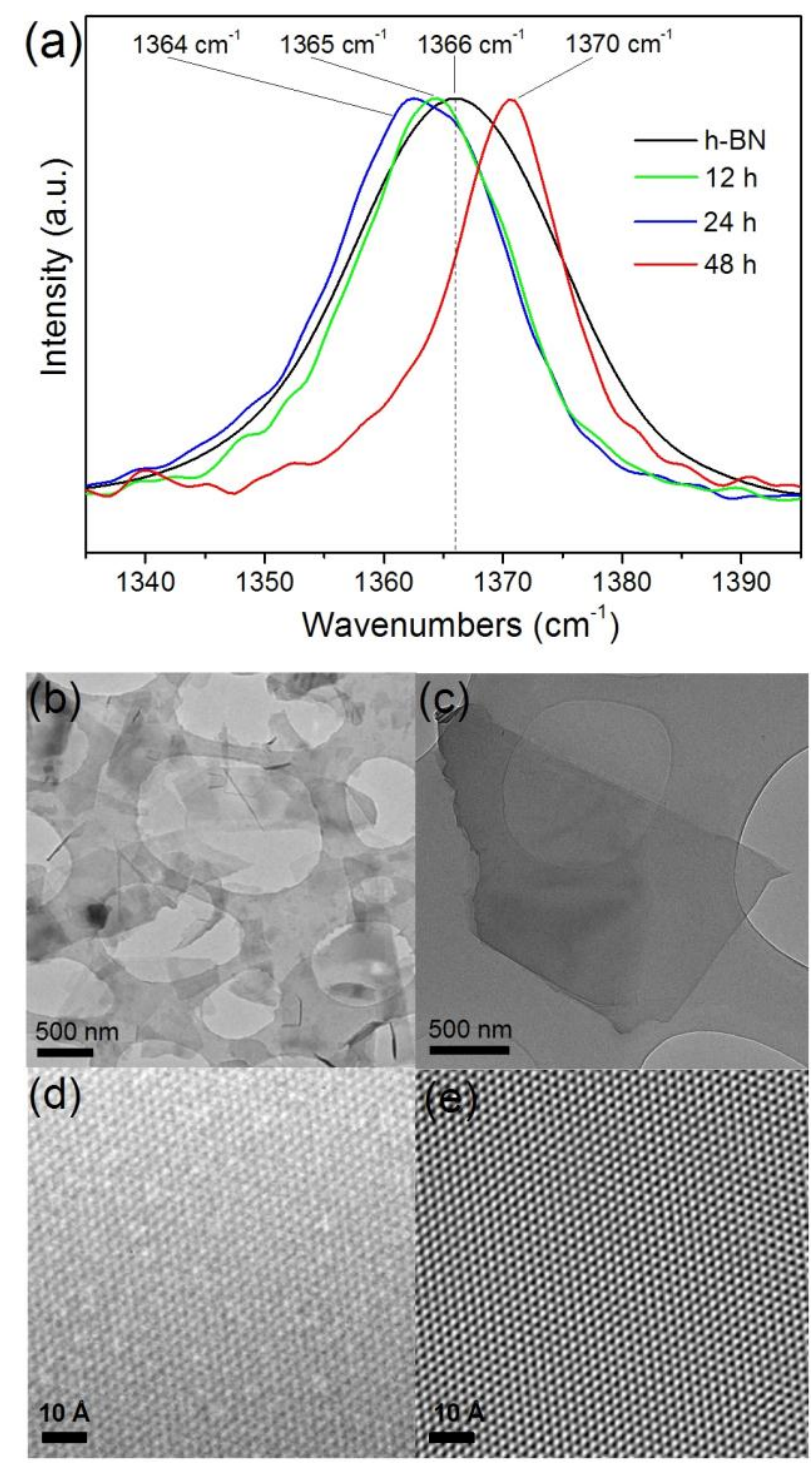

Figure 1. (a) Raman spectra of h-BN during exfoliation procedure at 12,24 and $48 \mathrm{~h}$. Inset shows $\mathrm{E}_{2 \mathrm{~g}}$ mode shift from $1366 \mathrm{~cm}^{-1}$ in bulk hBN during exfoliation. (b) TEM image showing exfoliated BNNSs following exfoliation protocol. (c) TEM image of single exfoliated BNNS on holey carbon grid indicating lateral dimensions of 2-3 $\mu \mathrm{m}$. (d) HRTEM image of the planar lattice structure of an exfoliated BNNS. (e) spatially-filtered image of Figure $2 \mathrm{~d}$ showing the hexagonal lattice structure.

The exfoliation of the BNNSs was also characterized using HRTEM, firstly to ensure that the procedure had produced a large fraction of mono- and few-layer sheets and secondly to ensure that the structural integrity of the nanosheets remained intact. Figure 1 shows representative HRTEM images using a low-voltage aberration corrected microscope of b) exfoliated BNNSs of lateral dimensions of the order of 2-3 $\mu \mathrm{m}, \mathrm{c}$ ) an individual BNNS. Figure 1d shows a typical unfiltered HRTEM image of a BNNS, the spatially filtered image of Figure 1d is shown as Figure 
1e. The TEM images show that the integrity of the h-BN lattice remains intact and is without defective holes or dislocations and that the exfoliation procedure produces BNNSs which are separated from large bulk h-BN crystallites. This in turn renders the exfoliated BNNSs available for reactive chemistry in solution. Additional TEM images and statistics are provided in the Supporting Information (Figure S2) which confirms the exfoliation protocol to produce mono- and few-layer BNNSs.

To functionalize the exfoliated BNNSs with oxygen radicals, exfoliated BNNS material was sonicated directly off a filter membrane into the clear TBP liquid in a round bottomed flask. The translucent milky white dispersion of BNNSs was then transferred to the Teflon inner jacket of a High Pressure Reactor Vessel (Autoclave) and heated to $120{ }^{\circ} \mathrm{C}$ for $12 \mathrm{~h}$ to thermolyze the TBP and generate the reactive oxygen radicals in the presence of the exfoliated nanosheets. The reaction pathway for the tert-butoxy radical functionalization of the BNNSs is illustrated in Scheme 2.

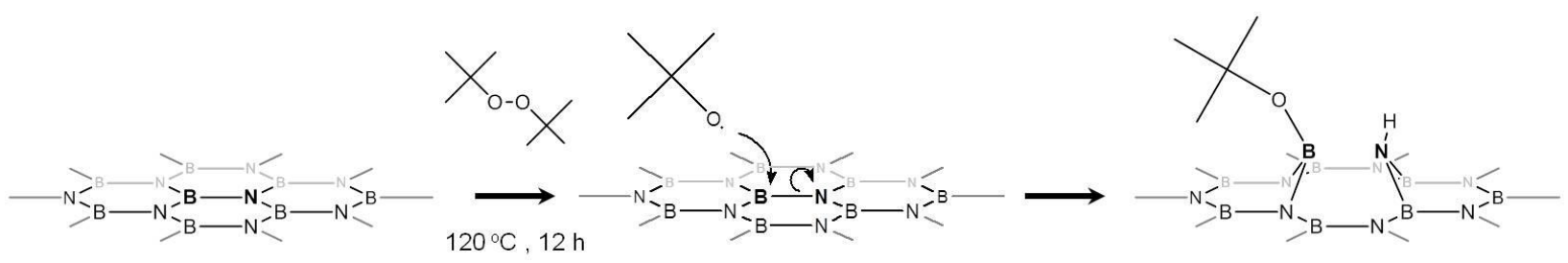

Scheme 2. Tert-butoxy radical functionalization of BNNSs via boronate ester formation.

As oxygen radicals are generated in the presence of BNNSs, the reaction proceeds by the attack of the reactive oxygen radical on the electron deficient boron atom. The oxidative attack on the boron atom to form the boronate ester must occur with concomitant cleavage of the in-plane B-N bond to satisfy the valence conditions. We speculate that the $\mathrm{N}$ atom would be protonated by dissolved moisture or protons generated by radical to radical reaction in the system. Consequently, the nitrogen atom/amine would be expected to become dislocated out of the planar $\mathrm{sp}^{2}$ lattice as its hybridization is modified to the tetrahedral $\mathrm{sp}^{3}$ configuration by the functionalization.

Following the reaction, the reactor vessel was allowed to cool to room temperature and was opened to reveal a yellow-orange cloudy dispersion of h-BN material which gave off a sweet odour with a hint of acetic acid. Filtration of the liquid using a $20 \mathrm{~nm}$ Alumina membrane filter allowed the separation of a clear yellow-orange liquid byproduct from the white h-BN material. The presence of moisture or protons either reacting with $\mathrm{h}-\mathrm{BN}$ during the reaction or upon exposure to air was confirmed by the analysis of the by-product using FTIR (Supporting Information, Figure S5). Identification of a large carbonyl band at $1710 \mathrm{~cm}^{-1}$, in addition to an $-\mathrm{OH}$ stretch at 3470 $\mathrm{cm}^{-1}$ and a broad C-O band in the 1000-1200 $\mathrm{cm}^{-1}$ region indicates the presence of carboxyl and hydroxyl groups in the by-product. The quenching of tert-butoxy radicals is expected to occur by radical-radical reaction in the absence of moisture and/or by moisture upon filtration, and exposure to air. Therefore dissolved protons/water may yield carboxyl and hydroxyl containing molecules such as tert-butanol and tert-butanoic acid. In view of this it is expected that protons would be available to quench dislocated $\mathrm{N}$ atoms in the $\mathrm{h}-\mathrm{BN}$ lattice to form secondary amines, thus supporting the proposed reaction mechanism. 
Recovery of the tert-butoxy-functionalized BNNSs (TB-BNNSs) involved an extensive washing procedure whereby, the BNNS material was filtered and washed using multiple solvents, dispersed by sonication and refiltered and re-washed to ensure that no starter material or by-products remained in the BNNS material.

UV-Vis Spectroscopy was used to determine the changes in the absorption/scattering behaviour of the BNNSs following the functionalization procedure. This was done by analyzing the optical absorption of dispersed, exfoliated BNNSs and functionalized TB-BNNSs in propanol. Both pristine-BNNSs and TB-BNNSs were prepared identically using the exfoliation protocol previously described. Exfoliated pristine-BNNSs were found to exhibit an absorbance value of 72 absorbance units $/ \mathrm{m}$ at $\lambda=300 \mathrm{~nm}$ (Figure 2). Following the determination of the mass of the exfoliated material, as described in the experimental section, the dispersed concentration was determined to be 0.10 $\mathrm{mgmL}^{-1}$. This allowed the calculation of the extinction coefficient to be $720 \mathrm{mLmg}^{-1} \mathrm{~m}^{-1}$. This value is in good agreement with our previous work. ${ }^{10 a}$ TB-functionalized-BNNSs (TB-BNNSs) were found to exhibit a markedly higher absorbance than that of the pristine-BNNSs (Figure 2), with both spectra exhibiting similar profiles indicating the scattering of the BNNSs in solution. A measured concentration of $0.122 \mathrm{mgmL}^{-1}$ (from filtration and weighing) corresponded to an absorbance value of 89 absorbance units $/ \mathrm{m}(\lambda=300 \mathrm{~nm})$. This corresponds to an extinction coefficient of $730 \mathrm{mLmg}^{-1} \mathrm{~m}^{-1}$. We speculate that the $\sim 23 \%$ increase in mass of solubilized BNNSs following functionalization indicates that organic groups grafted to the surface of the BNNSs give greater compatibility with the solvent. In this case, the aliphatic tert-butoxy functional groups significantly improve the solubility of BNNSs in propanol and therefore a better dispersion of the BNNSs is observed.

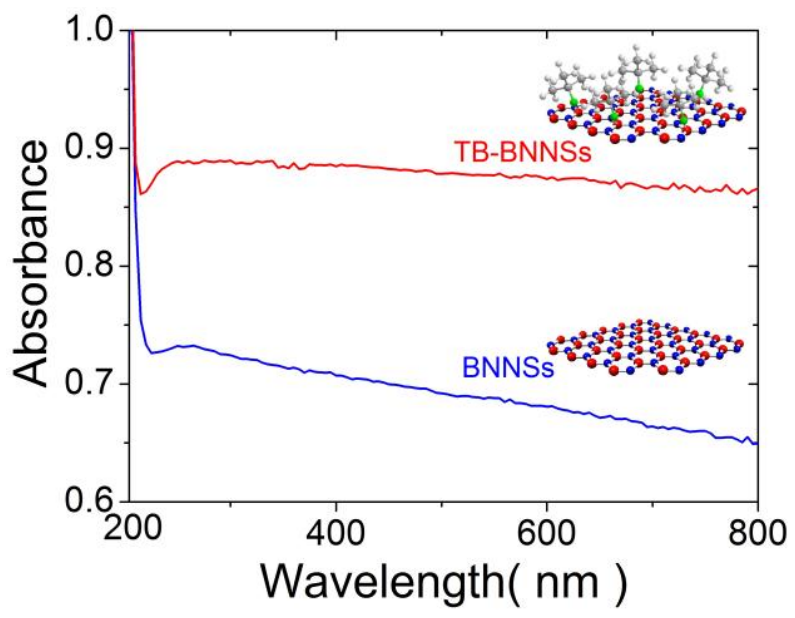

Figure 2. (a) UV-Vis spectra of pristine-BNNSs and TB-

BNNSs in isopropanol.

${ }^{1} \mathrm{H}-\mathrm{NMR}$ was also used to characterize tert-butoxy groups grafted to the surface of the BNNSs. In DMSO- $\mathrm{d}_{6}$, the free TBP molecule exhibits a single peak at $1.21 \mathrm{ppm}$ which corresponds to the 18 equivalent tert-butyl protons, Figure 3a. The spectrum of TB-BNNSs in DMSO- $\mathrm{d}_{6}$ exhibits two broadened bands at 1.13 and 0.73 ppm, 
corresponding to the tert-butoxy protons (Figure 3b). Broadening of the peaks is attributed to the a combination of the effect of binding of the molecules to the suspended BNNS substrate, which is known to significantly attenuate the signal and also the inhomogeneity of the environment of the groups to the dislocated lattice at the point of binding. ${ }^{33}$ It has been proposed that B-N bonds will break to facilitate the functionalization, consequently it is expected that there is a spread in the environment of the dislocation when the adduct is grafted to the B atoms and the $\mathrm{N}$ atom is protonated and dislocated out of the lattice plane, thus yielding the broadened peaks. The two peaks in the spectrum of the TB-BNNSs, in comparison with the single peak for the free TBP molecule, is explained by the conformational change incurred by the TB groups covalently bound to the solid nanosheet substrate. Several authors have reported that the slow-rotation of tert-butyl groups due to conformational restriction account for a shift in the expected position of tert-butyl groups and is observed as two peaks. ${ }^{34 a, b}$ In this case, it is believed that this type of conformational inhomogeneity results in the observed peaks of the TB-BNNSs.

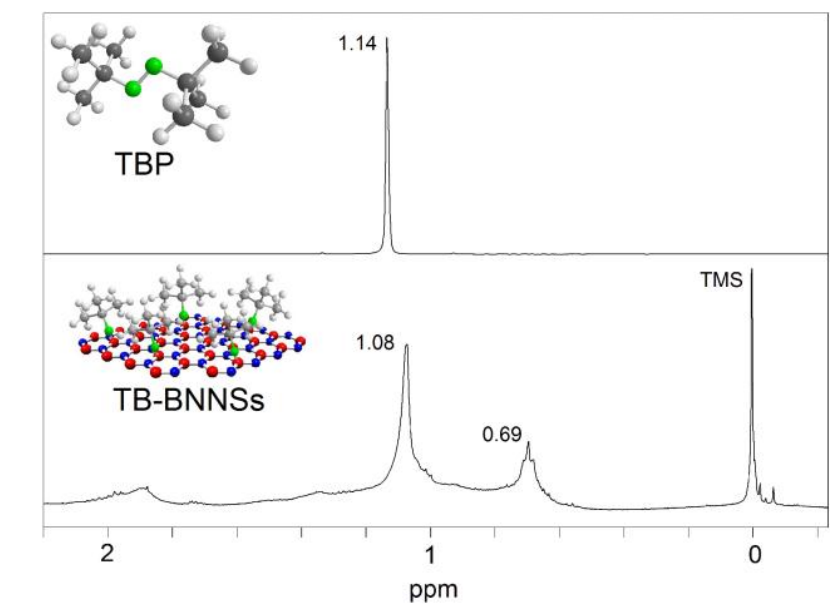

Figure 3. ${ }^{1} \mathrm{H}-\mathrm{NMR}$ spectrum of (a) tert-butylperoxide molecule (TBP), (b) tert-butoxy-functionalized-BNNSs (TB-BNNSs), both in DMSO-d6.

TGA analysis of the filtered, washed and dried TB-BNNS material was performed to quantify the organic fraction grafted to the BNNSs. Representative TGA traces of pristine-BNNSs (BNNSs) and TB-BNNSs are seen in Figure 4. A mass loss of $\sim 8 \%$ was recorded for TB-BNNSs, while the reference sample of pristine BNNSs remained constant until reaching temperatures of $\sim 850{ }^{\circ} \mathrm{C}$. Thereafter, for the pristine-BNNSs a slight mass gain $(\sim \leq 1 \%)$ was observed which is due to the formation of boron oxide, ${ }^{24}$ while the TB-BNNSs continued to lose mass until the temperature limit of the instrument was reached. This indicated a significant decrease in mass between $200-400{ }^{\circ} \mathrm{C}$ attributed to the loss of covalently grafted organic tert-butoxy groups. The onset of significant mass loss above 200 ${ }^{\circ} \mathrm{C}$ indicates the covalent nature of the species lost and precludes any residual solvents used during sample preparation. We interpret the continued mass loss above $800{ }^{\circ} \mathrm{C}$ as the restricted removal of the organic fraction from nanosheets which are re-stacked into layered bulk-phase material upon drying and results in the release of residual functional groups at higher temperatures $\left(>800{ }^{\circ} \mathrm{C}\right)$. We expect oxidation of the sample in the same manner as the pristine BNNSs and do not believe that the functionalization procedure prevents oxidation, rather, the oxidation mass gain is just off-set against a continued mass loss. This means that the observed mass loss of $\sim 8 \%$ 
may slightly under-report the maximum organic fraction. Secondly, the loss of $\sim 8 \%$, taking the mass of the tertbutoxy groups into account, yields a functionalization of $\sim 4$ at $\%$ of boron atoms in the lattice. It is calculated that along a sheet edge that there are 4 boron atoms every nanometer, and that the exposed sheet-edge boron atoms account for $0.044 \%$ of the total atoms in the nanosheets. Thus, the TGA analysis yielding $~ 4 \%$ of boron atoms functionalized represents two orders of magnitude difference over simple edge functionalization. This implies that functionalization of the centre of the nanosheets must occur and supports the assertion of the oxygen radical functionalization mechanism proposed.

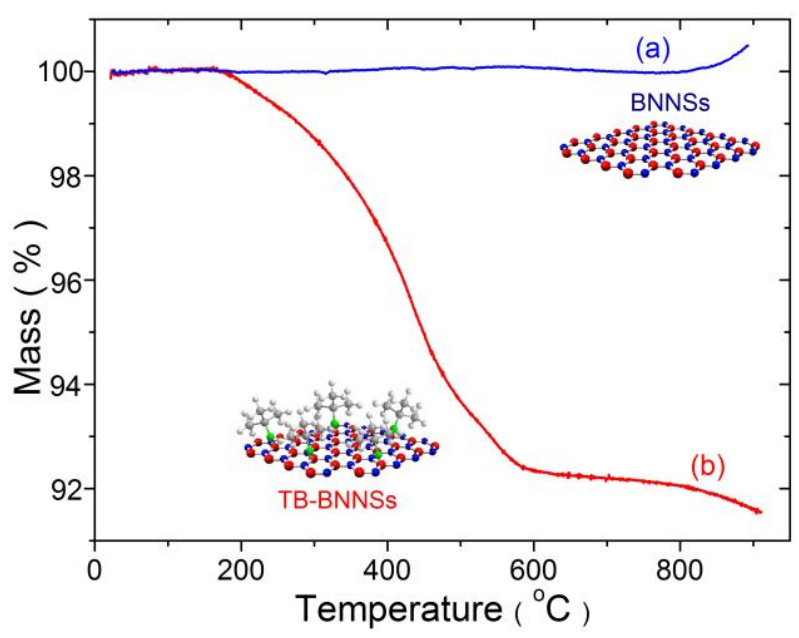

Figure 4. TGA traces of (a) pristine-BNNSs indicating a stable profile until oxidation above $800{ }^{\circ} \mathrm{C}$, (b) the profile of TB-BNNSs indicating substantial mass loss between $200-600{ }^{\circ} \mathrm{C}$ corresponding to organic grafted material.

The tert-butoxy radical functionalization of the BNNSs was followed by a strongly oxidizing treatment to remove the organic functionalization from the surface of the nanosheets. It is known that boronate esters are kinetically vulnerable to attack from water and undergo hydrolysis under ambient conditions to form the corresponding alcohols. ${ }^{35}$ Furthermore, the use of potent oxidants such as peroxides readily oxidizes all types of boronic acids and their boronate esters to yield corresponding alcohols. ${ }^{36}$ In view of this, we employ piranha solution $\left(\mathrm{H}_{2} \mathrm{O}_{2} / \mathrm{H}_{2} \mathrm{SO}_{4}\right)$, as a potent oxidizer to hydrolyze tert-butoxy groups grafted to the BNNSs to yield hydroxyl groups at the originally modified B atoms, thus hydroxyl-functionalized BNNSs (OH-BNNSs) as depicted in Scheme 3.

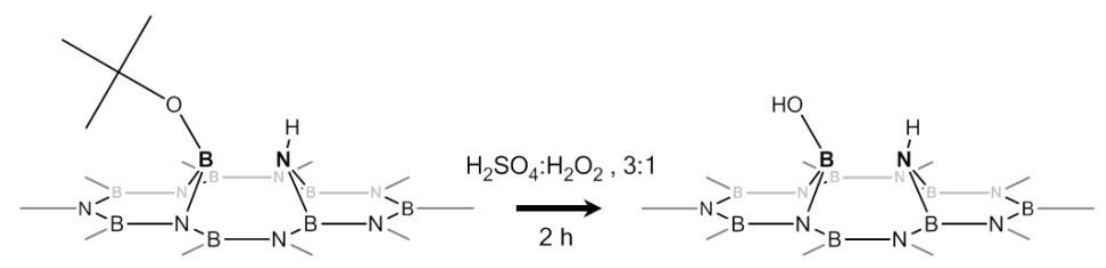


Scheme 3. Piranha solution de-functionalization of tert-butoxy-functionalized BNNSs to yield hydroxyl-functionalized BNNSs (OH-BNNSs).

TB-BNNSs material which was filtered, washed and dried following the functionalization procedure was dispersed by sonication in concentrated sulphuric acid. Hydrogen peroxide was added to the readily dispersed BNNSs to make up the 'piranha solution'. The potent oxidizing solution de-functionalizes the TB-BNNSs by hydrolysis of the boronate ester linkages to yield the B-OH hydroxyl groups. Following the oxidative procedure, extensive washing and filtration ensured the OH-BNNS product material was of the highest possible purity. The action of piranha solution results in carbon species being released as $\mathrm{CO}_{2}$ and thus precludes any residual organic contaminants. Notably, during the filtration procedure, as water was added to the filtration funnel, the BNNS material was immediately observed to disperse in solution suggesting the hydrophilic property of the OH-BNNSs (Supporting information Figure S4).

Optical absorption spectroscopy was carried out in order to investigate the change in solubility of the OH-BNNSs relative to pristine-BNNSs. Since h-BN typically exhibits limited solubility in water and is known to exhibit superhydrophobic behaviour, ${ }^{29 \mathrm{c}}$ the dispersion of OH-BNNSs in water serves to demonstrate the modification of the h-BN surface chemistry by the introduction of hydrophilic hydroxyl functional groups. ${ }^{10 a, 17,18}$ As before, preparation of identical pristine-BNNSs and OH-BNNSs samples for UV-Vis analysis involved the dispersion of a known mass of the material in solution by bath-sonication $(48 \mathrm{~h})$ followed by centrifugation $(1500 \mathrm{rpm}, 45 \mathrm{~min})$ to remove any aggregated material. The suspended material was allowed to equilibrate by standing for a further $24 \mathrm{~h}$ before analysis. The solvent was water and the initial concentration of the BNNSs was $1 \mathrm{mg} / \mathrm{mL}$. UV-Vis spectra of the OH-BNNSs and pristine-BNNS in water are shown in Figure 5. Pristine-BNNSs exhibit a scattering profile in solution while the spectrum of $\mathrm{OH}-\mathrm{BNNSs}$ has significantly higher absorbance and exhibit a modified profile suggesting that the OH-BNNSs have a substantially different solubilization in water due to surface hydroxyl functional groups. The inset (a) in Figure 5 shows a photograph of water, BNNSs and OH-BNNSs (left to right) and clearly shows the enhanced solubility of the OH-BNNSs.

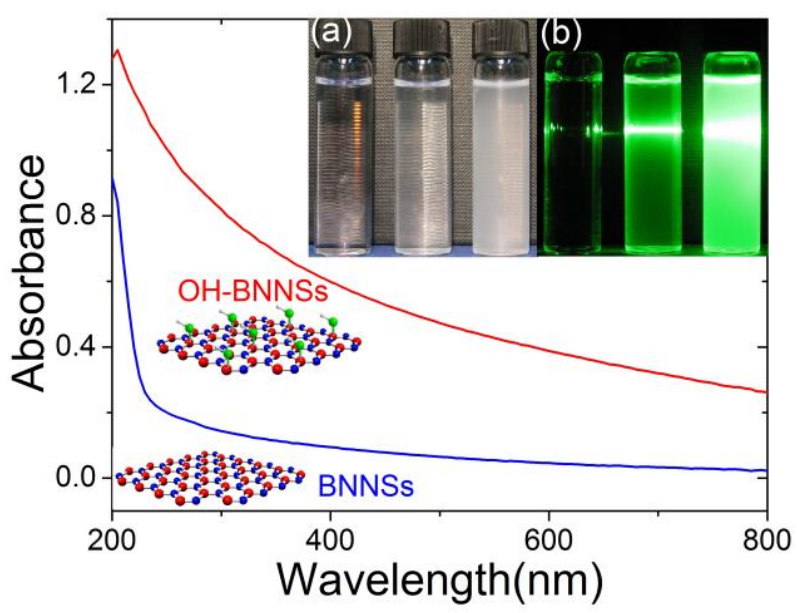


Figure 5. UV-Vis spectra of pristine-BNNSs and $\mathrm{OH}-\mathrm{BNNSs}$ in

water. With inset showing photographs of water, and pristine-

BNNSs and $\mathrm{OH}-\mathrm{BNNSs}$ in water.

To determine the precise mass of the solubilized material the solutions were filtered, dried and weighed. The concentration of the OH-BNNSs was found to be $0.107 \mathrm{mgmL}^{-1}$, while the pristine-BNNSs was $0.022 \mathrm{mgmL}^{-1}$. The five-fold difference in concentration is attributed to the modified surface chemistry of the OH-BNNSs which render the nanosheets water soluble due to the polar hydroxyl functional groups. This analysis also gives evidence for the validation of the proposed reaction mechanism whereby radical functionalization results in indiscriminate functionalization and not simple at edge sites. To further demonstrate the enhanced scattering behaviour of the $\mathrm{OH}-$ BNNSs relative to pristine-BNNSs the samples were irradiated with a $532 \mathrm{~nm}$ green laser. The Tyndall effect is indicative of the scattering effect of the nanosheets in solution, and it is clearly seen that the OH-BNNSs scatter the light to a much greater extent due to the mass of BNNSs effectively dispersed in solution, Figure 5 inset (b) showing water, BNNSs and OH-BNNSs from left to right.

It is known that h-BN exhibits superhydrophobicity in the bulk phase and at substrates; ${ }^{17,18,29 \mathrm{c}}$ however it has also been recently reported that using strong ultrasonication ${ }^{25}$ and ball-milling techniques ${ }^{37}$ have produced defect functionalization at edges and dislocations induced by the mechanical destruction of the h-BN lattice yielding a degree of water solubility. Therefore it is expected that upon exfoliation and perhaps scission of BNNSs fragments in water, that the sheets are slightly soluble, which we observe here. This is significant, as despite the identical preparation of the BNNS samples in this work, and even if the edges of the sheets had been functionalized via hydrolysis from the sonication procedure, OH-BNNSs are solubilized to a much greater extent, which is also supported by the TGA analysis indicating functional groups are grafted to the BNNSs. This suggests that the centre regions of the h-BN lattice of the functionalized BNNSs are chemically modified with groups which show greater compatibility to water than the pristine material and substantiate the envisaged functionalization by oxygen radicals.

FTIR was used in to characterize the covalent functionalization of the BNNSs, firstly, following the reaction procedure to introduce tert-butoxy groups to the surface of the BNNSs and secondly, following the oxidative procedure to oxidize the tert-butoxy groups to hydroxyl functional groups. Prior to the functionalization procedure, the spectrum of pristine exfoliated BNNS's exhibits only the characteristic in-plane B-N stretching vibration at 1366 $\mathrm{cm}^{-1}$ and out-of-plane bending vibration at $816 \mathrm{~cm}^{-1}$ respectively, Figure $6 \mathrm{a} .{ }^{38}$ Importantly, absent in the spectrum of the BNNSs are bands due to hydroxyl, amino or borane groups at defect sites or edges either following synthesis or following extended sonication involved in the exfoliation procedure. These groups would be expected in the FTIR spectrum at 3300, 3400 and at $2500 \mathrm{~cm}^{-1}$ if present. Characterization of the di-tert-butylperoxide (TBP) molecule prior to the functionalization of the BNNSs, shows that the spectrum of the molecule is dominated by the principle vibrational modes of the tert-butyl groups, the $\mathrm{C}-\mathrm{O}$ and the peroxide O-O, Figure 6b. The asymmetric and symmetric stretching of the methyl groups are observed at $2977 \mathrm{~cm}^{-1}$ and $2932 \mathrm{~cm}^{-1}$ respectively, while the corresponding bending vibrations are observed at $1475 \mathrm{~cm}^{-1}$ and $1375 \mathrm{~cm}^{-1}$. Skeletal C-C bands are evident at 1244 $\mathrm{cm}^{-1}$ and $750 \mathrm{~cm}^{-1}$, while $\mathrm{C}-\mathrm{O}$ stretch and O-O vibrational modes are observed as intense bands at $1191 \mathrm{~cm}^{-1}$ and 
$876 \mathrm{~cm}^{-1}$. Following thermolysis of the TBP in the presence of BNNSs, the spectrum of the tert-butoxyfunctionalized BNNSs (TB-BNNSs) is significantly modified from that of the pristine BNNSs, multiple bands are now evident in the spectrum of the TB-BNNSs consistent with the vibrations of the TBP molecule, Figure 6c. Bands at $2981 \mathrm{~cm}^{-1}$ and $2889 \mathrm{~cm}^{-1}$ are assigned to the asymmetric $\mathrm{C}-\mathrm{H}$ and symmetric $\mathrm{C}-\mathrm{H}$ modes of the tert-butyl groups, which are broadened relative to those in the free molecule indicating a perturbed vibrational freedom consistent with binding to a substrate. ${ }^{33}$ The B-N in-plane vibrational modes found at $1366 \mathrm{~cm}^{-1}$ in the pristine material is now shifted up-field by $17 \mathrm{~cm}^{-1}$ to $1383 \mathrm{~cm}^{-1}$ following tert-butoxy functionalization, indicating a substantial modification to the lattice vibrations due to the grafting of the bulky tert-butyl substituent's. The out-of-plane B-N mode is found at $816 \mathrm{~cm}^{-1}$ as in the pristine material, which indicates a retention of the overall structural integrity of the $\mathrm{sp}^{2}$ lattice. The $\mathrm{C}-\mathrm{H}$ bend of the tert-butyl groups is observed at $1356 \mathrm{~cm}^{-1}$, while the $\mathrm{C}_{-} \mathrm{CH}_{3}$ band is found at $1270 \mathrm{~cm}^{-1}$. Most significantly for evidence of covalent functionalization of the BNNSs, the appearance of bands at $1154 \mathrm{~cm}^{-1}$ and $1080 \mathrm{~cm}^{-1}$ are assigned to the $\mathrm{C}-\mathrm{O}$ and $\mathrm{B}-\mathrm{O}$ bands of the boronate ester. ${ }^{39}$ The shift in the C-O band from $1191 \mathrm{~cm}^{-1}$ in the free TBP molecule to $1154 \mathrm{~cm}^{-1}$ confirms the change from C-O-O to C-O-B of the boronate ester. The absence of a peroxide $\mathrm{O}-\mathrm{O}$ band in the spectrum at $876 \mathrm{~cm}^{-1}$ confirms thermolysis of the TBP molecules has occurred to generate tert-butoxy radicals and precludes any suggestion of residual starter TBP molecules. The appearance of a further band at $952 \mathrm{~cm}^{-1}$ is assigned to the out-of-plane B-O mode. This indicates the formation of the boronate ester distorting B atoms from the B-N sp ${ }^{2}$ lattice plane of the BNNSs, as suggested by the proposed reaction mechanism. This band strongly corroborates the evidence for boronate ester formation.

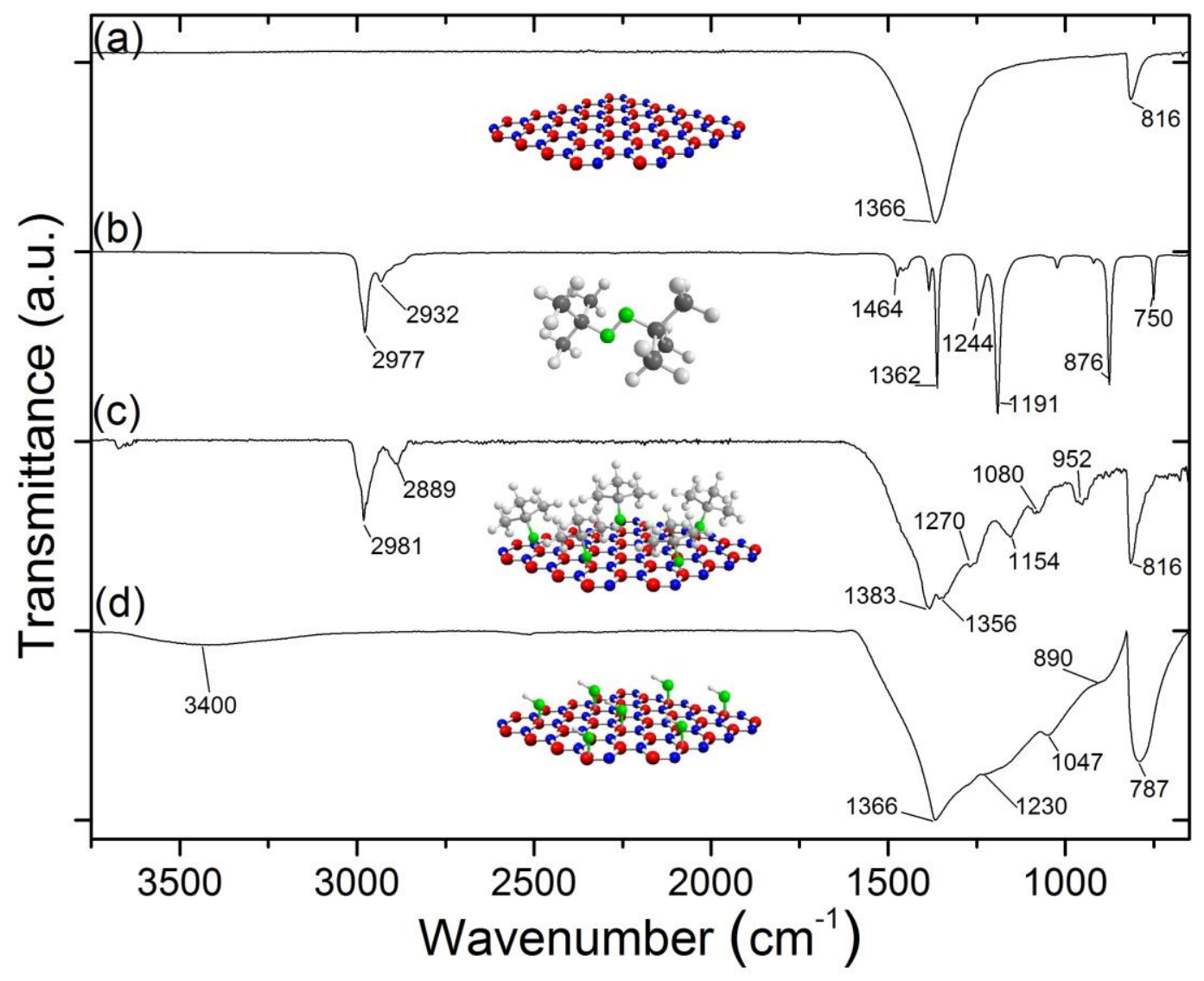


Figure 6. FTIR spectra of (a) pristine BNNSs, (b) tert-butylperoxide molecule, (c) tertbutoxy-functionalized-BNNSs (TB-BNNSs), (d) hydroxyl-functionalized-BNNSs (OH-

BNNSs).

An important feature from the FTIR analysis is the characterization of strong organic features relative to the signature of the underlying BNNSs. This is encouraging as we believe functionalization of the central h-BN lattice and not simply edge and defect functionalization has occurred. This is supported by the large shift in the in-plane mode of the BNNSs from $1366 \mathrm{~cm}^{-1}$ to $1383 \mathrm{~cm}^{-1}$ upon functionalization indicating that the single atom sheets are particularly sensitive to the grafting of adducts, particularly the bulky tert-butyl groups, Figure 6c.

The second phase of the functionalization procedure involves the hydrolysis of the surface-bound tert-butoxy groups using piranha solution to yield hydroxyl groups bound to the boron atoms and removal of the tert-butoxy bands. The spectrum of the hydroxyl-functionalized-BNNSs (OH-BNNSs) is shown in Figure 6d. The spectrum of OH-BNNSs (Figure 6d) is markedly different from that of the TB-BNNSs (Figure 6c). Following the oxidation of the surface bound tert-butoxy groups, the tert-butoxy methyl C-H stretching at $2981 \mathrm{~cm}^{-1}$ and $2889 \mathrm{~cm}^{-1}$ are absent and are replaced by the hydroxyl $\mathrm{O}-\mathrm{H}$ stretching band at $3400 \mathrm{~cm}^{-1}$. Water contamination which might affect this region of the spectrum is precluded as all samples for FTIR analysis were prepared by drying under vacuum using Schlenk apparatus for several days and no other bands were observed in the $1500-1800 \mathrm{~cm}^{-1}$ region. Bands that had been associated with the tert-butyl groups, the $\mathrm{C}-\mathrm{H}$ bending at $1356 \mathrm{~cm}^{-1}$ and $\mathrm{C}_{-} \mathrm{CH}_{3}$ at $1270 \mathrm{~cm}^{-1}$ and the $\mathrm{C}-\mathrm{O}$ stretch at $1154 \mathrm{~cm}^{-1}$ are now absent. Prominent bands at $1230 \mathrm{~cm}^{-1}$ and $1047 \mathrm{~cm}^{-1}$ are assigned to the in-plane bending of B$\mathrm{OH}$ and the symmetric B-O stretching (v1) band, while the shoulder at approximately $890 \mathrm{~cm}^{-1}$ represents the B-O$\mathrm{H}$ out-of-plane bending confirming the presence of the B-OH functional groups. ${ }^{40 a, \mathrm{~b}}$ The observed bands for the B$\mathrm{OH}$ stretching at $3400 \mathrm{~cm}^{-1}$ and the $\mathrm{B}-\mathrm{O}$ in-plane bending at $1230 \mathrm{~cm}^{-1}$ are in agreement with a recent theoretical study on Band-Gap engineering as a function of functionalization of h-BN sheets with -OH groups by Das et al. ${ }^{21}$ In this work the B-OH stretching band is predicted at $3430 \mathrm{~cm}^{-1}$ while the $\mathrm{B}-\mathrm{O}$ in-plane bend is predicted at $1240 \mathrm{~cm}^{-1}$.

The h-BN in-plane and out-of-plane modes at $1366 \mathrm{~cm}^{-1}$ and $787 \mathrm{~cm}^{-1}$ have broadened substantially and have shifted by $17 \mathrm{~cm}^{-1}$ and $29 \mathrm{~cm}^{-1}$ relative to the bands in the TB-BNNSs. We believe this shift is associated with the removal of the bulky tert-butoxy substituent's to form hydroxyl groups allowing vibration at lower frequencies and that the broadening represents an enhanced spread in the vibrational modes present after such an aggressive functionalization procedure. Undoubtedly, some degree of dislocation and disruption to the lattice will occur where boron atoms bond to hydroxyl functional groups to form boronic acid groups and $\mathrm{N}$ atoms must be dislocated to form amine groups. Both disruptions to the lattice would be expected to be out of the lattice plane and thus the vibrations of the B-N lattice would be affected, as we have observed. These shifts also suggest that the functionalization is covalent in nature as the vibrational bands of the BNNSs track directly as they shift up-field upon tert-butoxy functionalization to higher frequencies and back down-field to lower frequencies upon defunctionalization to the hydroxyl groups. We estimate that in order to cause such modifications that the degree of functionalization must be of a reasonable number, c.a. 4 at $\%$ based on the combined TGA, UV-Vis, and FTIR evidence. 
In summary, the covalent grafting of tert-butoxy groups to the surface of the BNNSs has been characterized via the formation of the C-O-B boronate ester and bands identifying the tert-butyl groups. Substantial shifts of bands in the free-molecule and the pristine-BNNSs as well as the absence of the peroxide group identify the covalent bond formation consistent with the TB-functionalization reaction envisaged. A subsequent reaction to remove tert-butoxy functionalization and yield $\mathrm{B}-\mathrm{OH}$ functionalization has been characterized. The absence of tert-butoxy bands coupled with the appearance of B-OH and B-O bands as well as shifts to the intrinsic B-N lattice vibrations support the assertion that the hydroxyl functionalization had occurred and that a substantial number of functional groups must have been created.

$\mathrm{X}$-ray photoelectron spectroscopy (XPS) was performed to characterize the increase in oxygen content in the BNNSs due to the presence of B-OH functional groups. The XPS spectrum of the pristine-BNNSs identified the B1s (Figure 7a) and N1s (Figure 7b) peaks at $190.9 \mathrm{eV}$ and $398.4 \mathrm{eV}$ respectively. Both values are in close agreement with values reported for single-layer h-BN and h-BN zones within mono-layer h-BNC at $190.9 \mathrm{eV}$ and $398.0 \mathrm{eV}$ respectively, and identifies the B-N bonding of the h- B-N phase. ${ }^{31 b, 41}$ The XPS survey spectra of pristine- and OHBNNSs are shown in Figure 7c, and compare the oxygen O1s peak of the samples, which is located at a binding energy of $532.9 \mathrm{eV}$ relative to the normalized N1s peak at $398.4 \mathrm{eV}$. In the spectrum of pristine-BNNSs, the O1s edge appears as a faint peak at $531.1 \mathrm{eV}$. This is assigned to background oxygen content of the sample, which may possibly be attributed to boric acid fragments present in the sample following sheet scission during exfoliation. Any residual reactant or by-product contamination during processing is precluded as both samples were prepared under identical conditions. Following the radical functionalization, the spectrum of the OH-BNNSs shows a large increase in the signal intensity of the O1s peak which is attributed to hydroxyl groups covalently grafted to the BNNSs. Analysis of the O1s/N1s intensity ratio indicates a value of 0.115 for the pristine BNNS sample, while the O1s/N1s ratio for the $\mathrm{OH}-\mathrm{BNNS}$ sample has increased over 6-fold to 0.731 . This is consistent with the change in the ratio of the integral of the O1s to the N1s peak, which indicates a 5.6 fold increase following functionalization. It is also worthy to note that the O1s peak of the pristine-BNNSs is centred at $532.9 \mathrm{eV}$ while for OH-BNNSs the O1s peak is centred at $531.1 \mathrm{eV}$. The $\sim 2 \mathrm{eV}$ difference is consistent with covalently bound $\mathrm{B}-\mathrm{OH}$ functional groups as distinct from oxygen contamination in the system. ${ }^{42}$ This confirms the addition of oxygen containing functional groups to the BNNSs as a result of the functionalization methodology. 

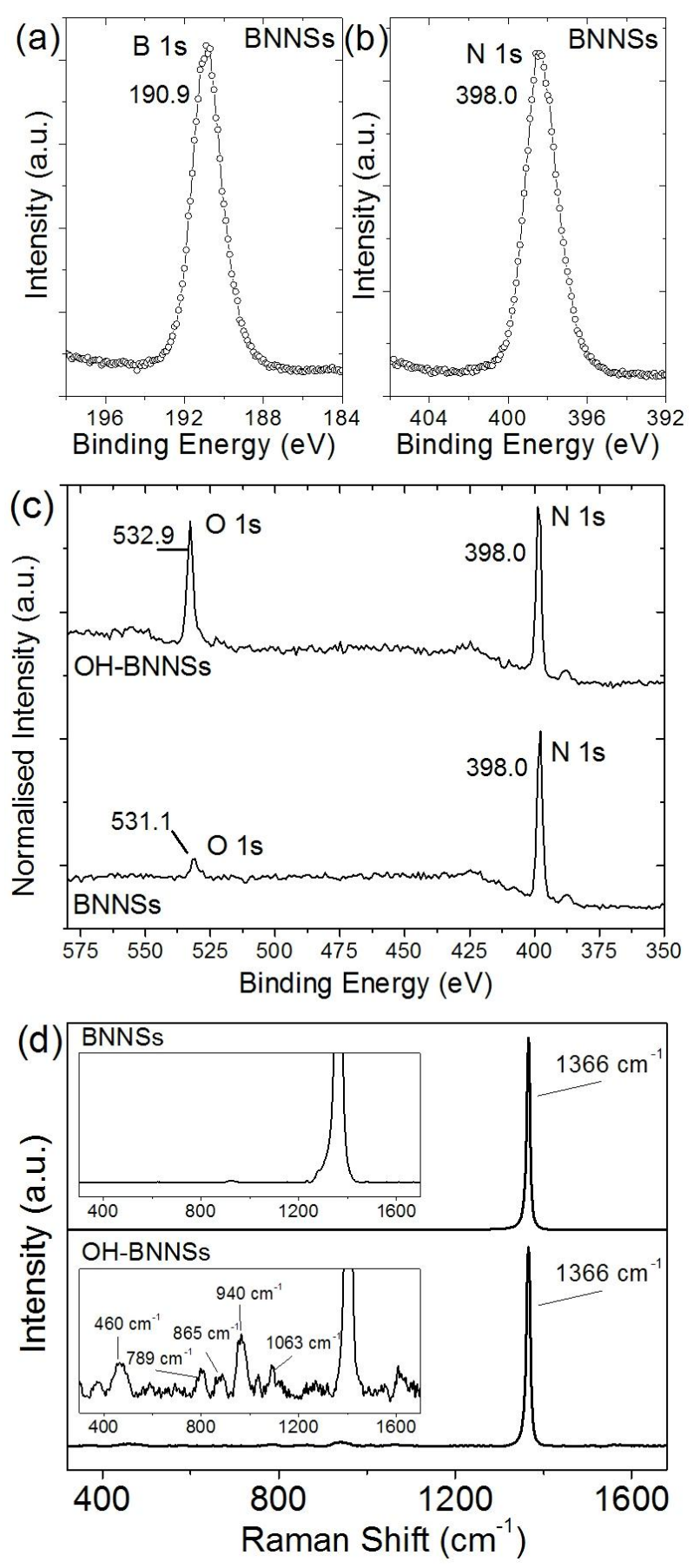

Figure 7. XPS spectra of BNNSs. (a) B 1s core level of BNNSs, (b) $\mathrm{N}$ 1s core level of BNNSs, both using fitting by Gaussian curves. (c) XPS wide spectrum of BNNSs and OH-BNNSs normalized to the $\mathrm{N} 1 \mathrm{~s}$ core level peak, and showing increased intensity of the $\mathrm{O}$ 1s core level for the OH-BNNSs following functionalization. (d) Raman spectra of BNNSs and OH-BNNSs normalized to the h-BN $E_{2 \mathrm{~g}}$ mode at $1366 \mathrm{~cm}^{-1}$. Shown in the inset are the full-scale expanded intensity spectra. 
Raman characterization of the OH-BNNSs was also performed in order to monitor the formation of B-OH groups following the oxidative functionalization treatment. Figure 7(d) shows the spectra of pristine-BNNSs (BNNSs) and OH-BNNSs respectively. The inset in Figure 7(d) shows the spectra of both BNNSs and OH-BNNSs normalized to the $\mathrm{E}_{2 \mathrm{G}} \mathrm{B}-\mathrm{N}$ vibrational mode at $1366 \mathrm{~cm}^{-1}$, which show a series of low intensity bands only in the case of the OHBNNSs. Expanding this region shows that the bands are located at $460 \mathrm{~cm}^{-1}, 789 \mathrm{~cm}^{-1}, 865 \mathrm{~cm}^{-1}, 940 \mathrm{~cm}^{-1}$ and 1063 $\mathrm{cm}^{-1}$. Comparison with values for free boric acid (principle bands at $500 \mathrm{~cm}^{-1}, 880 \mathrm{~cm}^{-1}$ and $1085 \mathrm{~cm}^{-1}$ ) suggest three of these OH-BNNS bands to be associated with the B-OH functional group. ${ }^{43-45}$ Shifts of $40 \mathrm{~cm}^{-1}, 15 \mathrm{~cm}^{-1}$, and 22 $\mathrm{cm}^{-1}$ are observed for the OH-BNNSs relative to the spectrum of free boric acid spectra. ${ }^{44,45}$ This is explained by effect of the bulk h-BN substrate on the vibration of the B-OH groups relative to the vibrational freedom of unbound molecules. ${ }^{33}$ The mode at $789 \mathrm{~cm}^{-1}$ in the spectrum of the OH-BNNSs is consistent with the Raman signature of an aryl-boronic acid and is not reported in Raman spectrum of free boric acid molecules. ${ }^{39,44}$ Considering the bonding structure of the h-BN lattice, and that the pseudo-delocalized ring structures may be extended out of the plane of the lattice following B atom functionalization and B-N bond cleavage, this band is assigned to the B-OH functional group. Finally we note that the band at $940 \mathrm{~cm}^{-1}$ is assigned to a second order mode of h-BN which is noted in studies by Loiseau and co-workers concerning h-BN and BNNTs. ${ }^{43}$

Following the functionalization procedure, TEM was used in order to assess the structural integrity of the BNNSs. Figure 8 shows representative TEM images of OH-BNNSs on a lacy carbon grid. It can be seen that the lateral dimensions of the nanosheets are of the order of 2-3 $\mu \mathrm{m}$ which is unchanged from that of the starting exfoliated BNNSs. The insets in the main image show a nanosheet protruding from its support and a high-resolution image indicating the undamaged structure of the h-BN lattice. Under constraints of the resolution used, it can be seen that the basic structure of the OH-BNNSs remains intact and that the functionalization has not broken up or created any large holes or fragmentation of the nanosheets. This is optimal for proposed utilization of functionalized BNNSs in mechanical, thermal or barrier composite applications.

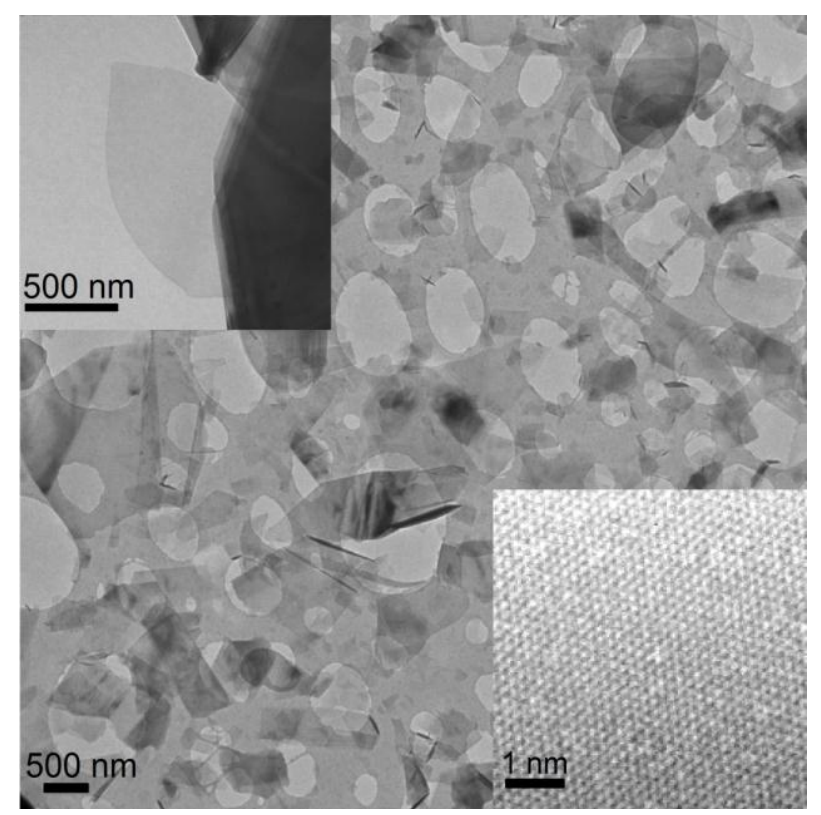


Figure 8. TEM image of OH-BNNSs on a lacy carbon grid.

Insets show an individual nanosheet at higher magnification

demonstrating the structural integrity following the

functionalization procedure.

The application of h-BN within polymer composites has attracted increasing attention recently on account of the extremely good mechanical properties of the material and due to its optical transparency. ${ }^{46}$ Using a white, insulating, high-strength nanosheet material over a black, conducting material such as graphene has particular advantages for high-strength-optically transparent composite materials which are not conductive and susceptive to charging. Wide application of composites based on Bisphenol-A epoxy resins, polycarbonate (PC), polymethylmethacryalate (PMMA), polyurethane (PU), polyvinylchloride (PVC) and polyethylene terephthalate (PET) and h-BN are envisaged if h-BN can be dispersed and optimally chemically interfaced within the intended host polymer matrix. With this as a motivating factor for this work, we have prepared BNNS-polymer composites of two major industrial polymers; polyvinyl alcohol (PVA) and polyurethane (PU) in order to firstly demonstrate chemical compatibilization with the polymer matrix and secondly enhance the mechanical properties of the polymer.

Polyvinyl alcohol:BNNS (BNNS:PVA) composites were formed by the preparation of aqueous dispersions of the polymer with and without the addition of $0.1 \mathrm{wt} \%$ water-soluble OH-BNNSs and pristine-BNNSs as the reference material. PVA as an excellent film forming and adhesive polymer has high tensile strength and flexibility and very good optical transparency. ${ }^{47}$ BNNS:PVA composites were prepared at the low mass fraction content of $0.1 \mathrm{wt} \%$ in order to both maintain the optical transparency of the polymer and to enhance the mechanical properties of the matrix. It is known that much of the recent literature concerning the preparation of nanosheet-composites, such as graphene and GO, have resulted in increases in Young's Modulus and tensile strength of the polymers while multiple examples document the reduction in the strain-to-break $\left(\varepsilon_{\mathrm{b}}\right)$ and toughness of the materials. ${ }^{48}$ It is not surprising that addition of a stiff material increases the Young's modulus of the matrix, however the reduction strain-to-break and toughness indicate that instead of enhancing all mechanical properties of the polymer, composites are more brittle relative to the pristine polymer. The addition of nanomaterials as filler in polymer composites often aggregate and act as defect points on account of their chemical incompatibility within the host polymer matrix. It has been demonstrated that optimal chemical integration of nanomaterials with the host matrix through chemical functionalization provides a means by which the interface between the nanomaterials and the polymer matrix is dictated by matched non-covalent or covalent chemical compatibility. ${ }^{49}$ Optimum dispersion of the filler material facilitates transfer of applied stress from the matrix to the filler yielding effective reinforcement. In view of this, here we chose polyvinyl alcohol (PVA) as its principle chemistry is associated with pendent hydroxyl groups from the carbon backbone and therefore has exactly matched chemical compatibility with the hydroxyl functionalized BNNSs (OH-BNNSs). BNNS:PVA composites were prepared with $\mathrm{OH}-\mathrm{BNNSs}(\mathrm{OH}-$ BNNS:PVA) and pristine-BNNSs (BNNS:PVA) by dispersing the BNNSs in aqueous solutions of PVA as described in the experimental section. Representative stress-strain curves for thin-film strips of PVA, BNNS:PVA and OHBNNS:PVA are shown in Figure 9a. The inset in Figure 9a shows the PVA, and BNNS:PVA and OH-BNNS:PVA composites on glass microscope slides and indicates the excellent transparency of the composite samples. Notably 
the slow evaporation of water to form the thin films on the glass slides yields perfectly clear samples and demonstrates the optical quality of the composites to be visible indistinguishable over the pristine PVA sample.

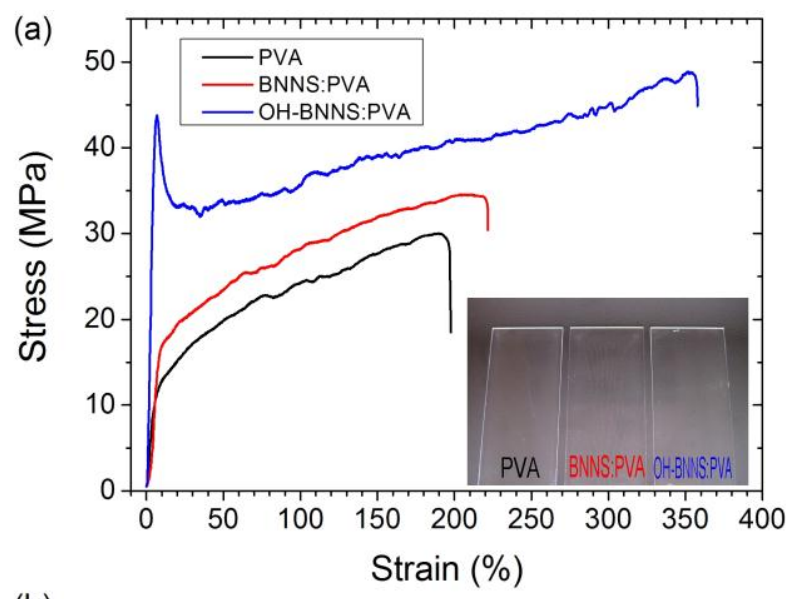

(b)

\begin{tabular}{|r|c|c|c|c|}
\hline & Y $(\mathrm{GPa})$ & UTS $(\mathrm{MPa})$ & $\varepsilon_{\mathrm{B}}(\%)$ & Toughness $\left(\mathrm{MJ} / \mathrm{m}^{3}\right)$ \\
\hline PVA & 0.378 & 29.5 & 199 & 115 \\
\hline BNNS:PVA & 0.452 & 34 & 223 & 165 \\
\hline OH-BNNS:PVA & 1.08 & 49 & 358 & 335 \\
\hline
\end{tabular}

Figure 9. (a) Representative stress-strain curves for PVA reference and BNNSs:PVA, OH-BNNSs:PVA composites. Inset shows the transparent samples on glass microscope slides. (b) Young's Modulus, Ultimate tensile strength, strain to break and toughness for the PVA reference and composites.

It can be seen that there is no substantial increase in Young's modulus of the BNNS:PVA (0.452 GPA) composite over the PVA reference ( $0.378 \mathrm{GPa}$ ) while the OH-BNNS:PVA composite displays a Young's modulus increase of over $200 \%$ to $1.08 \mathrm{GPa}$, Figure $9 \mathrm{~b}$. The ultimate tensile strength (UTS) increased marginally from $29.5 \mathrm{MPa}$ in the bulk polymer to 34.0 MPa in the BNNS:PVA composite but displayed an increase of $19.5 \mathrm{MPa}$ to $49.0 \mathrm{MPa}$ in the OH-BNNS:PVA composite. The strain-to break $\left(\varepsilon_{\mathrm{b}}\right)$ of the BNNS:PVA composite shows a slight increase $(223 \%)$ over the pristine polymer (199\%) which points to poor dispersion of BNNSs causing defect points within the matrix, while the OH-BNNS:PVA composite displays a large increase to $358 \%$. Correspondingly, toughness of the BNNS:PVA is increased marginally to $165.11 \mathrm{MJ} / \mathrm{m}^{3}$, while toughness of the OH-BNNS:PVA is increased by three times that of the pristine PVA $\left(115.8 \mathrm{MJ} / \mathrm{m}^{3}\right)$ to $335.38 \mathrm{MJ} / \mathrm{m}^{3}$. Notably the difference between the unfunctionalized- and functionalized-BNNSs indicates that the stress transfer mechanism in the OH-BNNS:PVA is markedly better. We assign this to improved dispersion of OH-BNNSs within the aqueous PVA solution and, upon drying, to the hydrogen-bonding cross-linking interactions of the BNNS-hydroxyl functionalities with the PVA chains at the interface between the materials (Supporting information, Scheme S1). This effect is identical to the way in which boric-acid is used to cross-link PVA and increase its mechanical properties. ${ }^{50}$ This effect has also been recently reported to facilitate the boric acid cross-linking of graphene-oxide (GO) nanosheets and is analogous to 
multiple reports concerning the formation of PVA-GO composites, in which increased mechanical properties are attributed to hydrogen bonding interactions. ${ }^{51 \mathrm{a-c}}$ In contrast, in the case of the pristine-BNNSs, poor dispersion and poor interfacial interaction are attributed to the mismatch in chemical compatibility between the materials.

The modified chemistry of the OH-BNNSs following functionalization is further characterized by clear differences in the mechanical properties of the composites and verifies that the chemistry of the nanosheets has a large influence on the ability of the nanosheets to interface with a polymer matrix. We extend the functionalization of BNNSs to demonstrate that the surface functional groups are amenable to chemical reaction and that by grafting a specific functional group to the BNNSs that the compatibilization of the BNNSs with a matrix of choice can be achieved. In the case of the PVA:OH-BNNSs, hydrogen-bonding mediates the interaction, we now demonstrate that covalent functionalization linkages are used to modify the surface chemistry and dictate the interaction with the polymer matrix. To do this, we use the formation of carbamate linkages with the hydroxyl functional groups on the $\mathrm{OH}$ BNNSs to form isocyanate/carbamate functionalized-BNNSs (iBNNSs) which can be compatibilized within a polyurethane matrix. This functionalization is identical to the methodology pioneered by Ruoff and co-workers which uses isocyanate-functionalization to functionalize alcohol groups in GO. ${ }^{52}$ It is also known that boric acid B$\mathrm{OH}$ groups form borocarbamates when reacted with isocyanates. ${ }^{53 \mathrm{a}, \mathrm{b}}$ Using this methodology, we demonstrate that this can be achieved for the white, insulating, and transparent structural analogue of graphene oxide, $\mathrm{OH}-\mathrm{BNNSs}$, to achieve optimal integration of h-BN within a polymer matrix. Isocyanate-functionalized-BNNSs (iBNNSs) were prepared by functionalization of OH-BNNSs with the diisocyanate; 1,6-hexamethylene diisocyanate (HMDI), via borocarbamate formation, as illustrated in Scheme 4.

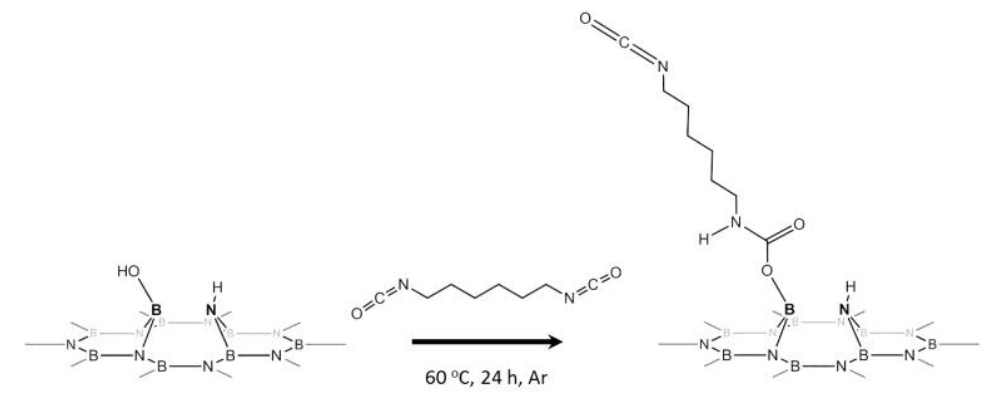

Scheme 4. 1,6-hexamethylene diisocyanate functionalization of OH-BNNSs via carbamate formation to yield isocyanate-functionalized BNNSs (iBNNSs).

Grafting of the diisocyanate molecule to the BNNSs provides functional compatibility of the carbamate group, and the alkyl chain within the polyurethane matrix. The terminal isocyanate group facilitates binding to hydroxyl or amine groups present at chain ends with the polyurethane matrix via carbamate and urea linkages respectively. Characterization of the iBNNSs was performed by FTIR to confirm the presence of the HMDI molecule at the surface of the BNNSs (Supporting Information, Figure S6). Bands due to alkyl methylene groups, terminal isocyanate group, identify the HMDI molecule while the appearance of a carbamate carbonyl band at $1733 \mathrm{~cm}^{-1}$ and amide II N-H band at $1635 \mathrm{~cm}^{-1}$, and boronate ester B-O-C identify the borocarbamate linkage to the OH-BNNSs hydroxyl groups. It is worthy to note that the formation of the borocarbamate cannot exist without the surface 
hydroxyl functional groups of the OH-BNNSs and offers further substantiation of the functionalization methodology.

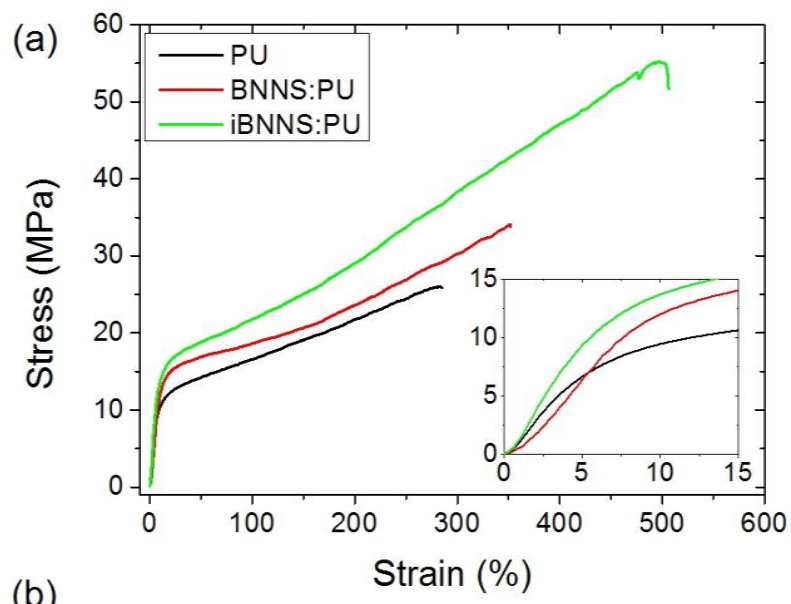

(b)

\begin{tabular}{|r|c|c|c|c|}
\hline & $Y(\mathrm{GPa})$ & UTS $(\mathrm{MPa})$ & $\varepsilon_{\mathrm{B}}(\%)$ & Toughness $\left(\mathrm{MJ} / \mathrm{m}^{3}\right)$ \\
\hline PU & 0.19 & 24.9 & 297 & 51 \\
\hline BNNS:PU & 0.215 & 33.7 & 354 & 80 \\
\hline iBNNS:PU & 0.229 & 54.2 & 513 & 170 \\
\hline
\end{tabular}

Figure 10. (a) Representative stress-strain curves for PU reference and BNNSs:PU, iBNNSs:PU composites. Inset showing initial onset of stress-strain curves between 0 15\%. (b) Young's Modulus, Ultimate tensile strength, strain to break and toughness for the PU reference and composites.

BNNS-Polyurethane (PU) composites; pristine-BNNSs:PU (BNNS:PU) and iBNNS:PU were formed by dispersion of the fillers within THF dispersions of PU. Thin films of the pristine PU and BNNS:PU, iBNNS:PU composites were formed on glass slides and samples prepared for mechanical testing, as described in the experimental section. Representative stress-strain curves for PU, BNNS:PU and iBNNS:PU are shown in Figure 10a. It can be seen from the inset in Figure 10a that the initial onset for the stress-strain curves between 0 and 15\% strain indicates distinct differences between pristine PU and the BNNS composites which is attributed to reinforcement from the nanosheets. On addition of BNNSs to PU the BNNS:PU composite shows an increase in Young's modulus from 0.19 GPa for the pristine PU to $0.215 \mathrm{GPa}$ for the BNNS:PU while the ultimate tensile strength (UTS) has increased 36\% from 24.9 $\mathrm{MPa}$ to $33.7 \mathrm{MPa}$, Figure 10b. The strain-to-break, $\varepsilon_{\mathrm{b}}$, increased from $297 \%$ to $354 \%$ while the overall toughness of the composite increased from $51 \mathrm{MJ} / \mathrm{m}^{3}$ to $80.15 \mathrm{MJ} / \mathrm{m}^{3}$. It is clear that the addition of the BNNSs to the PU matrix enhances the mechanical properties. In the case of the iBNNS:PU composite, the Young's modulus increased $21 \%$ from $0.19 \mathrm{GPa}$ to $0.229 \mathrm{GPa}$, while the UTS was increased by $116 \%$ from $24.9 \mathrm{MPa}$ to $54.2 \mathrm{MPa}$. The strain-to-break, $\varepsilon_{\mathrm{b}}$, increased from $297 \%$ to $513 \%$ while the corresponding toughness of the composite saw a three-fold increase from $51 \mathrm{MJ} / \mathrm{m}^{3}$ to $170.16 \mathrm{MJ} / \mathrm{m}^{3}$. It is clear that iBNNS significantly reinforces the PU matrix. Increases in both the strength and ductility of the PU are achieved by the addition of the iBNNS nanofiller, as well as large increases over the pristine-BNNSs-PU composite. We attribute these increases to the effective 
reinforcement of the PU matrix by the iBNNSs which are, in the case of the pristine-BNNSs, are well dispersed in the THF-PU solution during sample preparation and in the case of the iBNNSs, which are optimally dispersed in the polymer solution and also chemically matched and capable of binding with the PU matrix by the carbamate/isocyanate functionality (Supporting information, Scheme S2). While covalent binding of the iBNNSs to the PU matrix is indistinguishable using spectroscopy over the carbamate group formed by the diisocyanate functionalization of the BNNSs, the significant increase in ductility of the composite supports the assertion that covalent binding occurs at the interface between the materials. The difference in mechanical properties between pristine-BNNSs and iBNNSs substantiates the functionalization of the BNNSs by the described procedure and illustrates the importance of chemical compatibilization of filler materials within the polymer matrix. Notably, comparison between the BNNS reinforcement of PVA and PU highlights the incompatibility of the pristine-BNNSs with PVA which is consistent with their hydrophobic character while OH-BNNSs successfully reinforce the PVA matrix. In the case of the PU, pristine-BNNSs and iBNNSs reinforce the matrix due to the intrinsic compatibility of the BNNSs and the enhanced compatibility of iBNNSs to the THF/PU solution respectively. It is clear from these results that surface functionalization of the BNNSs mediates the interaction with the polymer matrix and that basic functionalization provides a means by which the chemistry of the nanosheets may be tuned to match an intended solvent, polymer or substrate.

\section{Conclusion.}

The solution phase oxygen radical functionalization of exfoliated BNNSs has been demonstrated using an organic peroxide as the source of oxygen radical species to achieve covalent grafting of tert-butoxy groups to BNNSs. The introduction of chemical functional groups to the surface of the BNNSs was utilized as a means by which hydroxyl functional groups could be generated upon removal of the organic groups using acid hydrolysis. The covalent functionalization of the BNNSs was confirmed using FTIR, ${ }^{1} \mathrm{H}-\mathrm{NMR}$, TGA, XPS and Raman spectroscopy. Most notably, FTIR, Raman, and X-Ray Photoelectron spectroscopy were used to specifically characterize the formation of the B-O bond. Further confirmation of the functionalization methodology was confirmed by subsequent derivatization between B-OH groups and di-isocyanate molecules via carbamate formation. Hydroxylated BNNSs (OH-BNNSs) and isocyanate-functionalized BNNSs (iBNNSs) were dispersed within polyvinyl alcohol (PVA) and polyurethane (PU) respectively to form nanocomposites. Mechanical analysis of the composites showed that there were significant enhancements of the mechanical properties and that the addition of chemically functionalized BNNS materials at a low mass fraction resulted in efficient polymer reinforcement. This is attributed to the homogeneous distribution and enhanced compatibility between the modified BNNSs and the polymer matrices. In the case of OH-BNNSs:PVA, hydroxyl to hydroxyl groups mediate the interaction via non-covalent hydrogen bonding interactions. In the case of the iBNNSs, the chemistry of the BNNSs are tuned to facilitate covalent bonding between the isocyanate functionalized iBNNSs and PU chains in addition to compatibility between the PU (alkyl polycarbamate) chains and the alkyl/carbamate functionalized BNNSs. Tuning the chemistry of nanofiller materials provides the basis for enhanced pathways for mechanisms of stress transfer in order to optimize the efficient reinforcement of next generation polymer nanocomposites. 
Acknowledgement. TS and JC would like to thank the European Research Council for funding through the grant SEMANTICS.

Supporting Information Available: Raman Spectroscopy and statistical analysis of exfoliation procedure, TEM analysis and statistical evaluation of exfoliation procedure, Optical photograph of water soluble OH-BNNSs, FTIR characterization of tert-butyl peroxide reaction by-product and isocyanate functionalized-BNNSs (iBNNSs), schematic illustration of OH-BNNS:PVA and iBNNS:PU composite interactions. This information is available free of charge via the internet at http://pubs.acs.org. 


\section{References}

$1 \quad$ Novoselov, K. S. Science 2004, 306, 666.

$2 \quad$ Park, S.; Ruoff, R. S. Nat. Nanotechnol. 2009, 4, 217.

3 Blake, P.; Brimicombe, P. D.; Nair, R. R.; Booth, T. J.; Jiang, D.; Schedin, F.; Ponomarenko, L. A.; Morozov, S. V.; Gleeson, H. F.; Hill, E. W.; Geim, A. K.; Novoselov, K. S. Nano Lett. 2008, 8, 1704. 3924.

5 Novoselov, K. S.; Jiang, D.; Schedin, F.; Booth, T. J.; Khotkevich, V. V.; Morozov, S. V.; Geim, A. K. Proc. Natl. Acad. Sci. U.S.A. 2005, 102, 10451-10453.

6 Radisavljevic, B.; Radenovic, A.; Brivio, J.; Giacometti, V.; Kis, A. Nat. Nanotechnol. 2011, 6, 147-150.

7 Dean, C. R.; Young, A. F.; Meric, I.; Lee, C.; Wang, L.; Sorgenfrei, S.; Watanabe, K.; Taniguchi, T.; Kim, P.; Shepard, K. L.; Hone, J. Nat. Nanotechnol. 2010, 5, 722-726.

8 (a) Hernandez, Y.; Nicolosi, V.; Lotya, M.; Blighe, F. M.; Sun, Z.; De, S.; McGovern, I. T.; Holland, B.; Byrne, M.; Gun'ko, Y. K.; Boland, J. J.; Niraj, P.; Duesberg, G.; Krishnamurthy, S.; Goodhue, R.; Hutchison, J.; Scardaci, V.; Ferrari, A. C.; Coleman, J. N. Nat. Nanotechnol. 2008, 3, 563-568. (b) Lotya, M.; Hernandez, Y.; King, P. J.; Smith, R. J.; Nicolosi, V.; Karlsson, L. S.; Blighe, F. M.; De, S.; Wang, Z.; McGovern, I. T.; Duesberg, G. S.; Coleman, J. N. J. Am. Chem. Soc. 2009, 131, 3611-3620.

$9 \quad$ Han, W. Q.; Wu, L.; Zhu, Y.; Wantanabe, K.; Taniguchi, I. Appl. Phys. Lett. 2008, 93, 223103.

(a) Coleman, J. N, et. al. Science 2011, 331, 568-571. (b) Stankovich, S.; Dikin, D. A.; Dommett, G. H. B.; Kohlhaas, K. M.; Zimney, E. J.; Stach, E. A.; Piner, R. D.; Nguyen, S. T.; Ruoff, R. S. Nature 2006, 442, 282-286. (c) Chang, K.; Chen, W.; Ma, L.; Li, H.; Huang, F.; Xu, Z.; Zhang, Q.; Lee, J.-Y. J. Mater. Chem. 2011, 21, 6251-6257. (d) Zhi, C.; Bando, Y.; Tang, C.; Kuwahara, H.; Golberg, D. Adv. Mater. 2009, 21, 2889-2893.

11 Sun, Z.; James, D. K.; Tour, J. M. J. Phys. Chem. Lett. 2011, 2, 2425-2432.

He, H.; Gao, C. Chem. Mater. 2010, 22, 5054-5064.

(a) Ramanathan, T.; Abdala, A. A.; Stankovich, S.; Dikin, D. A.; Herrera-Alonso, M.; Piner, R. D.; Adamson, D. H.; Schniepp, H. C.; Chen, X.; Ruoff, R. S.; Nguyen, S. T.; Aksay, I. A.; Prud'Homme, R. K.; Brinson, L. C. Nat. Nanotechnol. 2008, 3, 327-331. (b) Pan, Y.; Bao, H.; Sahoo, N. G.; Wu, T.; Li, L. Adv. Funct. Mater. 2011, 21, 2754-2763. 
Compton, O. C.; Nguyen, S. T. Small, 2010, 6, 711-723.

Arenal, R.; Blase, X.; Loiseau, A.; Adv. Phys. 2010, 59, 101-179.

Han, W.-Q. Anisotropic Hexagonal Boron-Nitride nanomaterials: Synthesis and Applications.

Nanotechnologies for the Life Sciences. Wiley 2010. Published online: 15 Oct. 2010.

Pakdel, A.; Zhi, C.; Bando, Y.; Nakayama, T.; Golberg, D. ACS Nano, 2011, 5, 6507-6515.

Yu, J.; Qin, L.; Hao, Y.; Kuang, S.; Bai, X.; Chong, Y.-M.; Zhang, W.; Wang, E. ACS Nano, 2010, 4, 414422 .

Itakura, A.; Tosa, M.; Ikeda, S.; Yoshihara, K. Vacuum, 1996, 47, 697-700.

Wu, J. C. S.; Lin, Z.-A.; Pan, J.-W.; Rei, M.-H. Appl. Cat. A: Gen. 2001, 219, 117-124.

Bhattacharya, A.; Bhattacharya, S.; Das, G. P. Phys. Rev. B, 2012, 85, 035415.

Lopez-Bezanilla, A.; Huang, J.; Terrones, H.; Sumpter, B. G. Nano Lett. 2011, 11, 3267-3273.

(a) Zhou, J.; Wang, Q.; Sun, Q.; Jena, P. Phys. Rev. B, 2010, 81, 085442. (b) Wu, X.-J.; Wu, M.-H.; Zeng, X. C. Front. Phys. China, 2009, 4, 367-372.

(a) Golberg, D.; Bando, Y.; Huang, Y.; Terao, T.; Mitome, M.; Tang, C.; Zhi, C. ACS Nano, 2010, 4, 29792993. (b) Han, W.-Q.; Mickelson, W.; Cumings, J.; Zettl, A. Appl. Phys. Lett. 2002, 81, 1110-1112. (c) Li, C.; Bando, Y.; Zhi, C.; Huang, Y.; Golberg, D. Nanotechnology, 2009, 20, 385707.

Lin, Y.; Williams, T. V.; Xu, T.-B.; Cao, W.; Elsayed-Ali, H. E.; Connell, J. W. J. Phys. Chem. C 2011, $115,2679-2685$.

May, P.; Khan, U.; O’Neill, A.; Coleman, J. N. J. Mater. Chem. 2012, 22, 1278-1282.

(a) Wang, W.; Bando, Y.; Zhi, C.; Fu, W.; Wang, E.; Golberg, D. J. Am. Chem. Soc. 2008, 130, 8144-8145.

(b) Zhi, C.; Bando, Y.; Tang, C. Golberg, D. J. Am. Chem. Soc. 2005, 127, 17144-17145. (c) Han, W.-Q.; Zettl, A. J. Am. Chem. Soc. 2003, 125, 2062-2063.

(a) Lin, Y.; Williams, T. V.; Connell, J. W. J. Phys. Chem. Lett. 2010, 1, 277-283. (b) Zhi, C.; Fu,W.; Wang, E.; Golberg, D. J. Am. Chem. Soc. 2005, 127, 15996-15997.

(c) Ikuno, T.; Sainsbury, T.; Okawa, D.; Frechet, J. M. J.; Zettl, A. Solid State Commun. 2007, 142, 643646. (d) Zhi, C. Y.; Bando, Y.; Terao, T.; Tang, C. C.; Kuwahara, H.; Golberg, D. Chem. Asian. J. 2009, 4, 1536-1540.

30 Nazarov, A. S.; Demin, V. N.; Grayfer, E. D.; Bulavchenko, A. I.; Arymbaeva, A. T.; Shin, H.-J.; Choi, J.Y.; Fedorov, V. E Chem. Asian. J. 2012, 7, 554-560. 
(a) Gorbachev, R. V.; Riaz, I.; Nair, R. R.; Jalil, R.; Britnell, L.; Belle, B. D.; Hill, E. W, Novoselov, K. S.; Watanabe, K.; Taniguchi, T.; Geim, A. K.; Blake, P. Small, 2011, 7, 465-468. (b) Song, L.; Ci, L.; Lu, H.; Sorokin, P. B.; Jin, C.; Ni, J.; Kvashnin, A. G.; Kvashnin, D. G.; Lou, J.; Yakobson, B. I.; Ajayan, P. M. Nano Lett. 2010, 10, 3209-3215.

Reich, S.; Ferrari, A. C.; Arenal, R.; Loiseau, A.; Bello, I.; Robertson, J. Phys. Rev. B 2005, 71, 205201.

Holzinger, M.; Vostrowsky, O.; Hirsch, A.; Hennrich, F.; Kappes, M.; Weiss, R.; Jellen, F. Angew. Chem. Int. Ed. 2001, 40, 4002 .

(a) Gill, G.; Pawar, D. M.; Noe, E. A. J. Org. Chem. 2005, 70, 10726-10731. (b) Rithner, C. D.;

Bushweller, C. H. J. Am. Chem. Soc. 1985, 107, 7823-7836.

(a) Haruta, R.; Ishiguro, M.; Ikeda, N.; Yamamoto, H. J. Am. Chem. Soc. 1982, 104, 7667-7669. (b)

Rousch, W. R.; Walts, A. G.; Hoong, L. K. J. Am. Chem. Soc. 1985, 107, 8186-8190.

Boronic Acids: Preparation and Applications in Organic Synthesis, Medicine and Materials, Second Ed.

Ed. D. G. Hall. 2011 Wiley-VCH Verlag GmbH \& Co. KGaA.

Lin, Y.; Williams, T. V.; Cao, W.; Elsayed-Ali, H. E.; Connell, J. W. J. Phys. Chem. C. 2010, 114, 1743417439.

Geick, R.; Perry, C. H.; Rupprecht, G.; Phys. Rev. 1966, 146, 543.

Infrared and Raman Characteristic Group Frequencies: Tables and Charts. G. Socrates, John Wiley and Sons Inc. 2004.

(a) Peak, D.; Luther, G. W, III; Sparks, D. L.; Geochimica et Cosmochimica Acta. 2003, 67, 2551-2560. (b) Zhu, D.; Jakovidis, G.; Bourgeois, L.; Bambery, K. Austr. Inst. Phys, $18^{\text {th }}$ National Congress, 2008, $119-$ 121.

Shi, Y.; Hamsen, C.; Jia, X.; Kim, K. K.; Reina, A.; Hofmann, M.; Hsu, A. L.;Zhang, K.; Li, H.; Juang, Z.Y.; Dresselhaus, M. S.; Li, L.-J.; Kong, J. Nano Lett. 2010, 10, 4134-4139.

Sachdev, H.; Müller, F.; Hüfner, S. Diamond Relat. Mater. 2010, 19, 1027-1033.

Arenal, R.; Ferrari, A. C.; Reich, S.; Wirtz, L.; Mevellec, J.-Y.; Lefrant, S.; Rubio, A.; Loiseau, A. Nano Lett. 2006, 6, 1812-1816.

Krishnan, K. Proc. Ind. Acad. Sci. 1963, A, 57, 103-108.

Erdemir, A.; Bindal, C.; Zuiker, C.; Savrun, E. Surf. Coat. Technol. 1996, 86-87, 507-510.

Zhi, C.; Bando, Y.; Tang, C.; Kuwahara, H.; Golberg, D. Adv. Mater. 2009, 21, 2889-2893. 
47 Brandrup, J. Polymer Handbook, $4^{\text {th }}$ Edition, Wiley 1999.

48 Potts, J. R.; Dreyer, D. R.; Bielawski, C. W.; Ruoff, R. S. Polymer 2011, 52, 5-25.

49 Coleman, J. N.; Khan, U.; Gun'ko, Y. K. Adv. Mater. 2006, 18, 689-706.

50 Tsukasa, M.; Yuuki, T.; Sachiko, A.; Takahiko, I.; Akie, H.; Keiko, E. Polymer, 2010, 51, 5539-5549.

51 Zhao, X.; Zhang, Q.; Hao, Y.; Li, Y.; Fang, Y.; Chen, D. Macromolecules, 2010, 43, 9411-9416.

52 Stankovich, S.; Piner, R. D.; Nguyen, S. T.; Ruoff, R. S. Carbon, 2006, 44, 3342-3347.

53 (a) Ruigh, W. L.; Dunnavant, W. R.; Gunderloy, F. C.; Steinberg, N. G.; Sedlak, M.; Olin, A. D. Adv.

Chem. 1961, 32, 27, 241-244. (b) Cragg, R. H.; Lappert, M. F. Boron-Nitrogen Chemistry, ACS: Advances in Chemistry, 1964, Vol. 42, 22, 220-226.

\section{Table of Contents Graphic:}

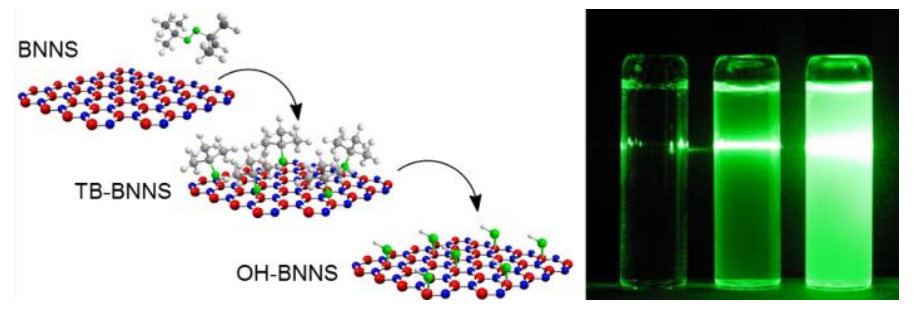

